\title{
A Multidimensional Spline Based Global Nonlinear Aerodynamic Model for the Cessna Citation II
}

\author{
C.C. de Visser, ${ }^{*}$ J.A. Mulder, ${ }^{\dagger}$ \\ Q.P. Chu ${ }^{\ddagger}$ \\ Delft University of Technology, Delft, The Netherlands
}

\begin{abstract}
A new method is proposed for the identification of global nonlinear models of aircraft non-dimensional force and moment coefficients. The method is based on a recent type of multivariate spline, the multivariate simplex spline, which can accurately approximate very large, scattered nonlinear datasets in any number of dimensions. The new identification method is used to identify a global nonlinear aerodynamic model of high dimensionality for the Cessna Citation II laboratory aircraft operated by the Delft University of Technology and the Netherlands National Aerospace Laboratory. The data used in the identification process consisted of millions of measurements and was accumulated during more than 250 flight test maneuvers with the laboratory aircraft. The resulting models for the aerodynamic force and moment coefficients are continuous analytical functions as they consist of sets of piecewise defined, multivariate polynomials. The identified models were validated using a subset of the flight data, with validation results showing a very close match between model and reality.
\end{abstract}

\section{Nomenclature}

$\bar{c} \quad=$ mean aerodynamic chord, $m$

$\hat{d} \quad=$ total number of polynomial terms in basis function

B $\quad$ global data location matrix

c $\quad$ global B-coefficient vector

D = global data sifting matrix

$\mathbf{H} \quad=$ smoothness matrix

$\mathbf{I} \quad=$ inertia matrix, $\mathrm{kg} \cdot \mathrm{m}^{2}$

$\mathbf{X}=$ regression matrix

$\mathbf{Y}=$ measurement vector

$\mathcal{T}=$ triangulation formed by a set of simplices

$A_{x}, A_{y}, A_{z}=$ specific forces along body $X / Y / Z$ axis, $\mathrm{m} / \mathrm{s}^{2}$

$a_{x}, a_{y}, a_{z}=$ kinematic accelerations along body $X / Y / Z$ axis, $m / s^{2}$

$b=$ barycentric coordinate

$B_{\kappa}^{d}(b)=$ individual polynomial basis function term of degree $d$

$C=$ general dimensionless coefficient,-

$c_{\kappa} \quad=$ individual B-coefficient

$d \quad=$ polynomial degree

$g \quad=$ gravity constant, $\mathrm{m} / \mathrm{s}^{2}$

$L, M, N=$ combined aerodynamic and thrust moments about the body $X / Y / Z$ axis, $N \cdot m$

${ }^{*}$ Ph.D. student, Control and Simulation Division, Faculty of Aerospace Engineering, P.O. Box 5058, 2600GB Delft, The Netherlands; c.c.devisser@tudelft.nl Student member AIAA.

${ }^{\dagger}$ Professor, Control and Simulation Division, Faculty of Aerospace Engineering, P.O. Box 5058, 2600GB Delft, The Netherlands; j.a.mulder@tudelft.nl Member AIAA.

${ }^{\ddagger}$ Associate Professor, Control and Simulation Division, Faculty of Aerospace Engineering, P.O. Box 5058, 2600GB Delft, The Netherlands; Q.P.Chu@tudelft.nl Member AIAA. 


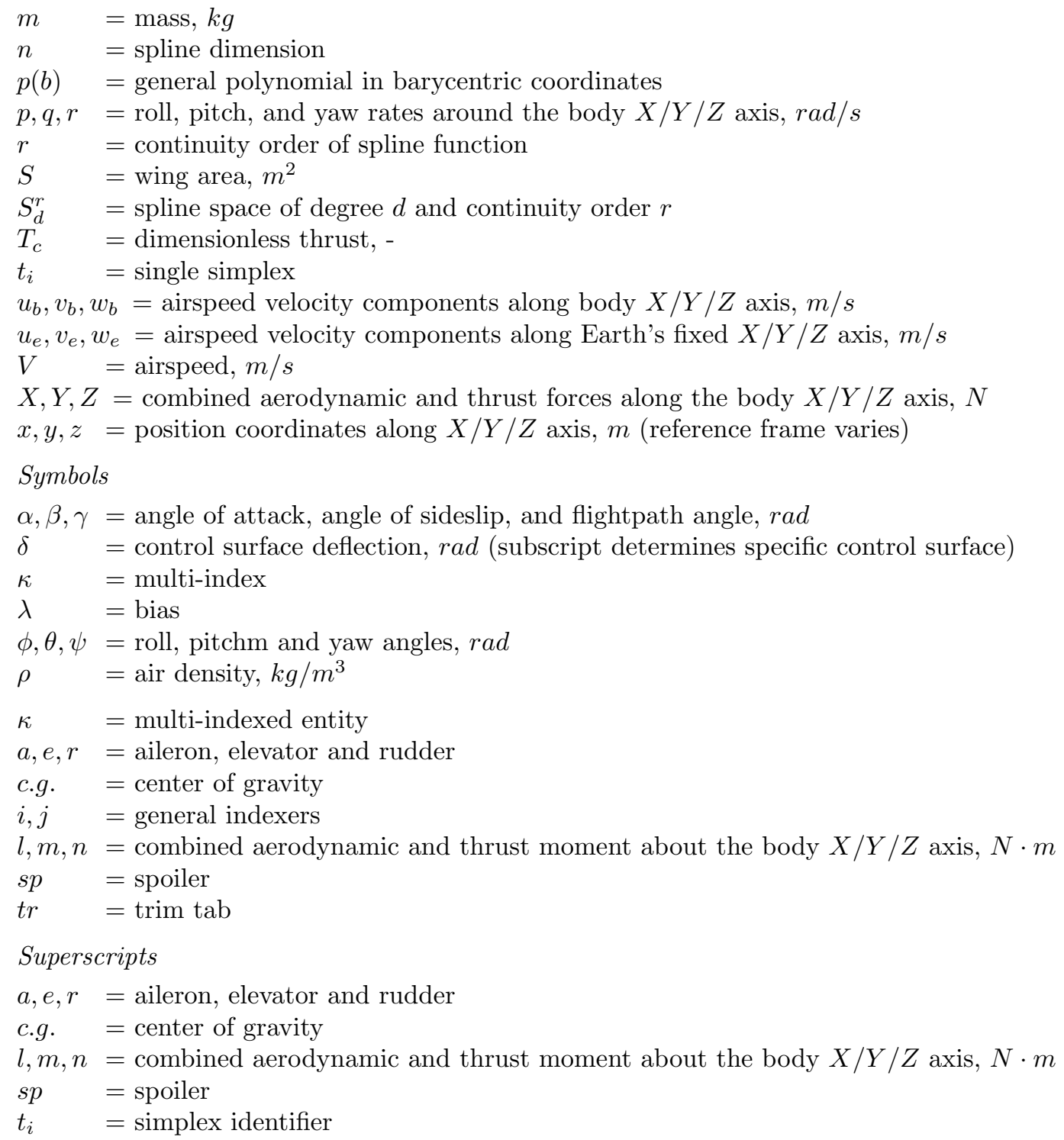

\section{Introduction}

Detailed aerodynamic models play a crucial role in the design of flight simulators as well as flight control systems. Currently, these models are in most cases implemented in the form of lookup tables or polynomial models. It is well known that both these model types have important and fundamental limitations. Lookup tables are used mostly in high performance (fighter) aircraft with large flight envelopes and non-linear aerodynamic characteristics, such as Eurofighter Typhoon. ${ }^{1,2}$ Updating, or expanding, an aerodynamic model based on lookup tables is a complex and time consuming task which must be performed offline. One of the reasons for the complexity of this task is the inherently discrete, gridded nature of data tables. ${ }^{3}$ Flight data is inherently scattered and will never completely match the grid points of the lookup tables. Therefore, some interpolation, or table superpositioning system must always be used to incorporate new data into the lookup tables.

The other model type used in aerodynamic models is the polynomial model, which has seen many uses over the past decades. ${ }^{4-9}$ Polynomial models assume some polynomial model structure for a force or moment coefficient. Parameter estimation techniques are then employed to estimate the parameters of the polynomials such that they, in some way, optimally fit a set of wind tunnel or flight test data. ${ }^{9-12}$ It is well known that polynomials have a limited approximation power, which is proportional with their polynomial degree. This in turn limits the application of polynomial based aerodynamic models to aircraft with small 
flight envelopes and limited nonlinear aerodynamics. More complex models can be created, however, by creating local polynomial models on partitions of the flight envelope. The set of local polynomial models can then be blended into a single smooth structure with for example fuzzy blending techniques or neural networks. ${ }^{13}$

Next to lookup tables and polynomial models, artificial neural networks can also be used to model aircraft aerodynamics. ${ }^{12,14,15}$ Neural networks are powerful nonlinear approximators, but suffer from a fundamental intransparency. While very useful for low to moderate complexity modeling tasks, their use inside flight control systems is limited as evident from their current absence in any such systems. Training a neural network is a computationally expensive process involving much trial and error tweaking. Additionally, the quality of a complex neural network is very hard to assure because of its black-box nature.

Many authors have therefore suggested the use of polynomial spline functions for fitting flight data. ${ }^{11,16-18}$ Spline functions are piecewise defined polynomials with a predefined continuity order between pieces. The approximation power of spline functions is proportional with the degree of the polynomial but also with the number and density of the polynomial pieces. Polynomial spline functions are therefore capable of fitting highly nonlinear datasets over large domains. Effectively, spline functions combine the best of two worlds in the sense that they combine the global nonlinear modeling capability of lookup tables with the analytic, continuous nature of polynomials.

While one-dimensional (i.e. univariate) spline theory is well known and developed, multi-dimensional (i.e. multivariate) spline theory is still an active research field. Many different multivariate spline types exist, such as thin plate splines ${ }^{19,20}$ and the well known and much used tensor product B-spline, ${ }^{21}$ and more recently, the multivariate simplex spline. In the past, multivariate tensor product splines have been successfully used to model aircraft aerodynamics. Smith, ${ }^{16}$ Klein $^{7,17}$ and Bruce ${ }^{18}$ used bivariate tensor product splines in a linear regression framework. Tensor product splines are, for fundamental mathematical reasons, defined on rectangular domains which greatly limits their flexibility. More importantly, it is well known that the multivariate tensor product spline is incapable of fitting scattered data, ${ }^{21,22}$ making it inadequate for the fitting of scattered flight test data.

The objective of this paper is the demonstration of a new method for aerodynamic model identification based on multivariate simplex splines. ${ }^{23-26}$ This new method has a high level of automation and can be used on very large datasets. The resulting aerodynamic model is a continuous analytical function which allows for local model modification without disrupting the global model structure.

At the center of the new identification method is a recent type of multivariate spline called the multivariate simplex spline. The multivariate simplex splines are capable of fitting nonlinear, multi-dimensional scattered data $^{27-29}$ and have an arbitrarily high approximation power. Simplex splines are not defined on rectangular domains and are therefore much more flexible than tensor product splines. Recently, a linear regression framework for multivariate simplex splines was introduced ${ }^{29}$ which allows the use of standard parameter estimation techniques such as Least Squares (LS) or Maximum Likelihood (ML) for estimating the parameters of the simplex spline polynomials. This new identification method was used to identify a global nonlinear aerodynamic model for the F-16 fighter aircraft ${ }^{30}$ based on simulated flight test data from a NASA subsonic wind tunnel model of the F-16. In this paper, the new identification method is used to identify a global aerodynamic model for the Cessna Citation II laboratory aircraft Fig. 1 (PH-LAB) based on flight data obtained during $300+$ maneuvers.

The Cessna Citation II laboratory aircraft is operated by the Delft University of Technology and the Netherlands National Aerospace Laboratory (NLR). The aircraft is a twin-jet business aircraft equipped with two Pratt \& Whitney JT15D-4 turbofan engines. The maximum operating altitude of the aircraft is $43,000 \mathrm{ft}$ and the maximum cruising speed is $385 \mathrm{kt}$. The aircraft is equipped with a state of the art flight test instrumentation system (FTIS) developed by the Delft University of Technology. At the heart of the FTIS system is a dSpace computer which is connected to an advanced suite of inertial, GPS, control input, and air data sensors Fig. 2. Additionally, the laboratory aircraft was recently equipped with a fly-by-wire (FBW) control system, allowing fully automatic execution of preprogrammed maneuvers.

\section{Preliminary on Multivariate Simplex Splines}

This section serves as a brief introduction on the mathematical theory of the multivariate simplex spline. For a more complete and in-depth coverage of the matter, we would like to refer to the work of Lai and Schumaker. ${ }^{26}$ The mathematical theory of the multivariate simplex spline is relatively recent, with de 


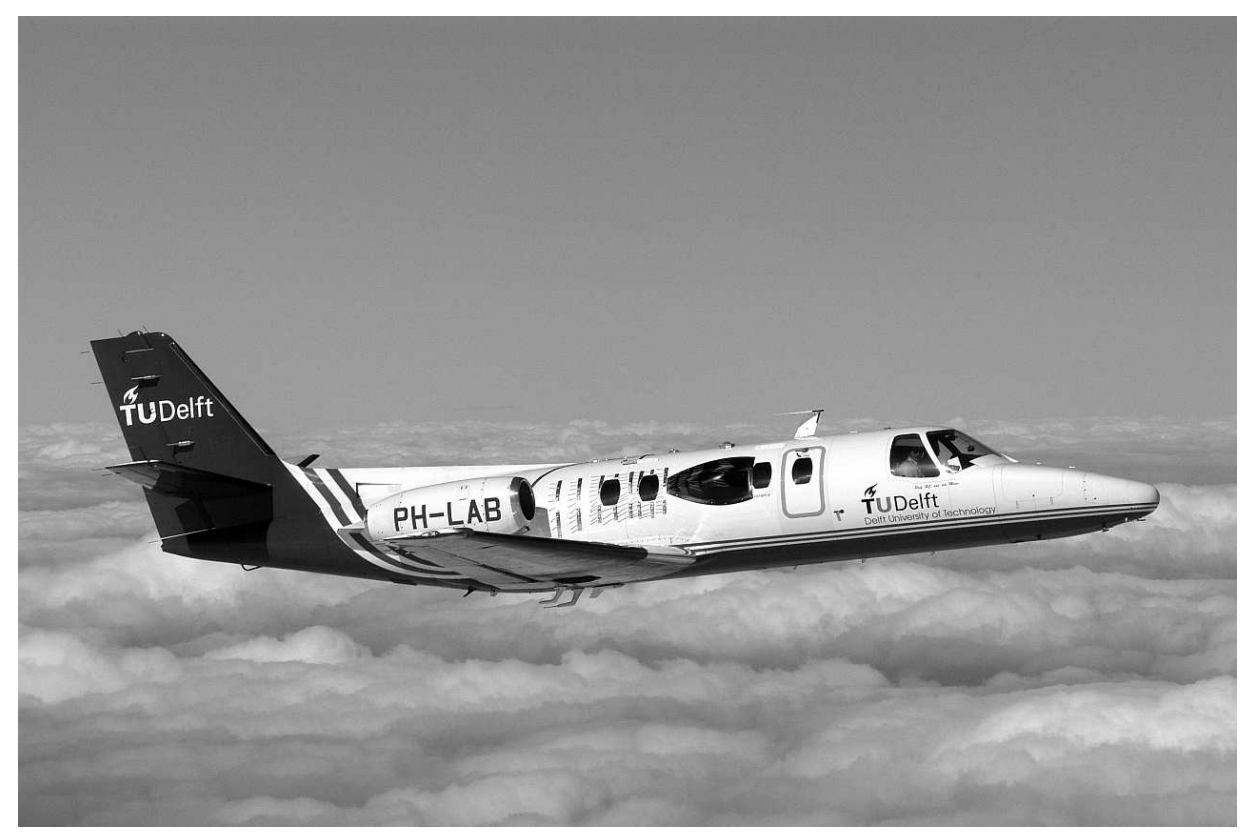

Fig. 1 Cessna Citation II laboratory aircraft

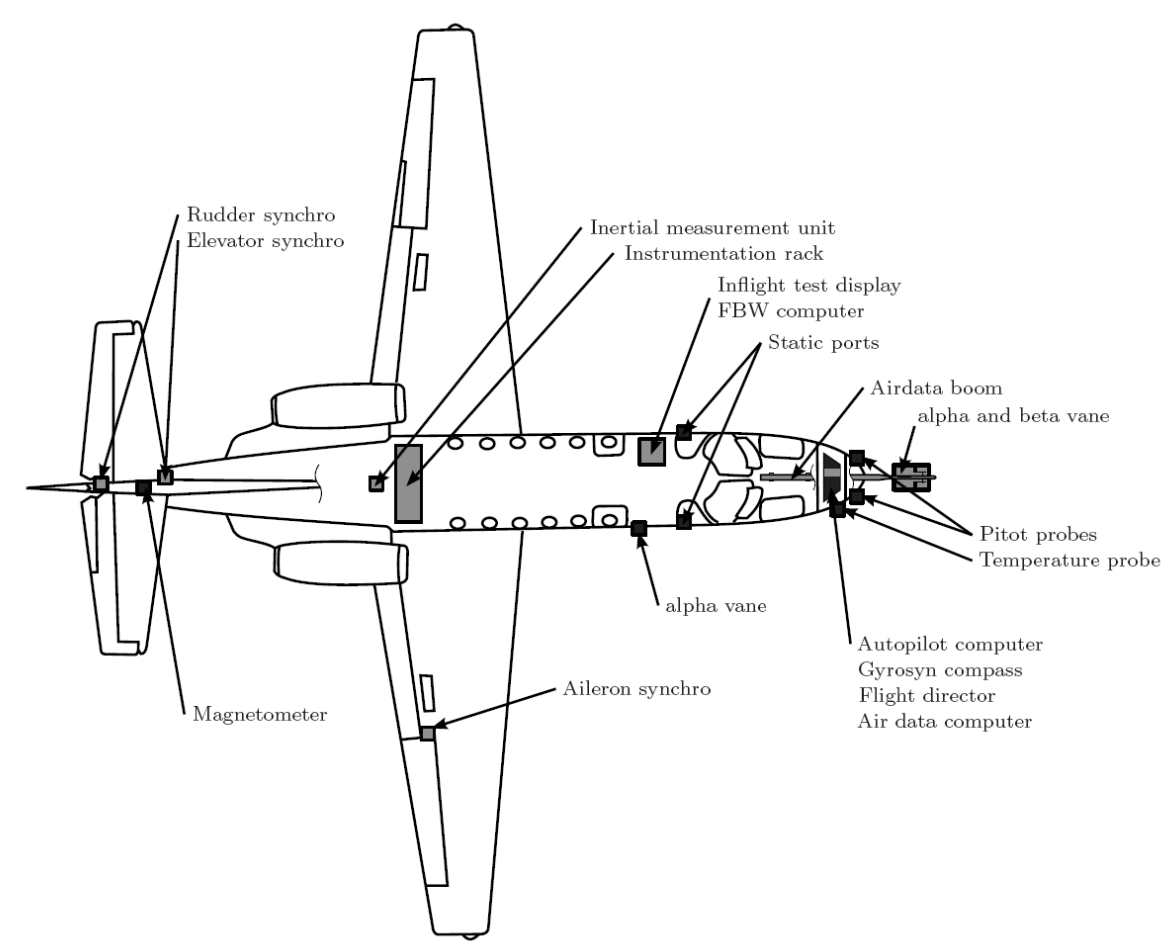

Fig. 2 Cessna Citation II laboratory aircraft flight test instrumentation system 
Boor, ${ }^{23}$ Lai and Schumaker ${ }^{24,26,31,32}$ and Awanou ${ }^{25,27}$ being important authors. The first practical scheme for scattered data approximation with multivariate simplex splines was published in $2005 .{ }^{27}$

Because the mathematical formalism of the multivariate simplex spline is equal in all dimensions we will often omit the term "'multivariate"' in the following.

\section{A. The Simplex and Barycentric Coordinates}

The individual spline pieces of the simplex spline are defined on simplices. A simplex is a geometric structure that provides a minimal, non-degenerate span of $n$-dimensional space. For example, the simplex of 2 dimensional space is the triangle and the simplex of 3-dimensional space the tetrahedron. Note that in the following we use ' $n$-simplex' as shorthand for 'the simplex of $n$-dimensional space'. A simplex is defined as follows. Let $V$ be a set of $n+1$ unique, non-degenerate, points in $n$-dimensional space:

$$
V:=\left\{v_{0}, v_{1}, \ldots, v_{n}\right\} \in \mathbb{R}^{n}
$$

Then the convex hull of $V$ is the $n$-simplex $t$ :

$$
t:=\langle V\rangle
$$

The boundary edges of a simplex are called facets. A facet of an $n$-simplex is a $(n-1)$-simplex by definition; it is constructed from all but one of the vertices of the $n$-simplex.

The simplex has its own local coordinate system in the form of the barycentric coordinate system. The barycentric coordinate system is instrumental in the definition of the stable local polynomial basis for the multivariate spline. The principle of barycentric coordinates is the following; every point $x=\left(x_{1}, x_{2}, \ldots, x_{n}\right)$ inside or outside the convex hull of a simplex $t$, with $t$ as in Eq. (2), can be described in terms of a unique weighted vector sum of the vertices of $t$. The barycentric coordinate $b(x)=\left(b_{0}, b_{1}, \ldots, b_{n}\right)$ of $x$ with respect to simplex $t$ are these vertex weights:

$$
x=\sum_{i=0}^{n} b_{i} v_{i}
$$

The barycentric coordinates are normalized, i.e.

$$
\sum_{i=0}^{n} b_{i}=1
$$

\section{B. Triangulations of Simplices}

A triangulation $\mathcal{T}$ is a special partitioning of a domain into a set of $J$ non-overlapping simplices.

$$
\mathcal{T}:=\bigcup\left\{t_{i}, i=0,1, \ldots, J\right\}
$$

In a valid triangulation simplices are not allowed inside the convex hull of other simplices:

$$
t_{i} \cap t_{j} \in\{\emptyset, \tilde{t}\}, \quad \forall t_{i}, t_{j} \in \mathcal{T}
$$

with $\tilde{t}$ a $k$-simplex with $0 \leq k \leq n-1$.

One of the most common triangulation methods is the Delaunay triangulation. Fig. 3 shows two Delaunay triangulations. In the left hand plot the Delaunay triangulation of 20 randomly distributed vertices in 2-D is shown. This triangulation consists of 31 triangles. In the right hand plot the Delaunay triangulation of 8 uniformly distributed vertices in $3-\mathrm{D}$ is shown. This triangulation consists of 6 tetrahedrons. It should be noted at this point, that creating a triangulation using the Delaunay algorithm does not always result in a well-defined triangulation, as has been pointed out in the literature ${ }^{33}$ and demonstrated in Fig. 4 . It is possible that so-called sliver simplices are produced, which are simplices which have a very large circum (hyper) sphere compared to their size. ${ }^{33}$ Polynomials defined on sliver simplices tend to be badly conditioned possibly leading to numerical instability, so their creation should be avoided as much as possible.

For our applications, we have developed a simple but powerful method for creating well defined triangulations suitable for use in aerodynamic model identification. This method does not use the Delaunay triangulation method, but instead fits a predefined number of (hyper) cubes inside the convex hull of a given dataset, see Fig. 5. The hypercubes themselves are then triangulated individually, resulting in a well defined triangulation such as that demonstrated in Fig. 5] 

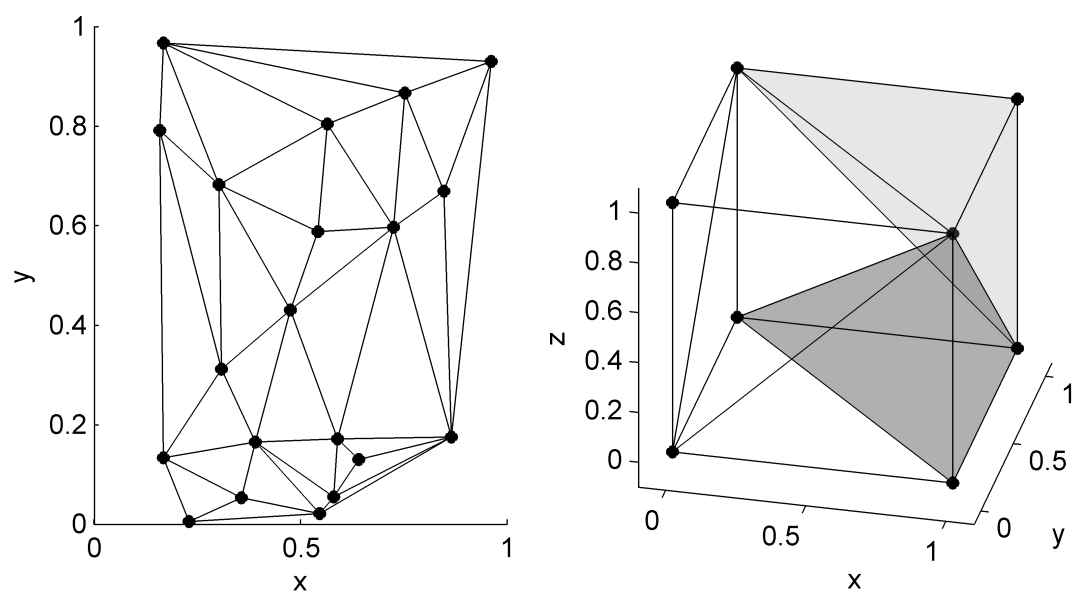

Fig. 3 2-D Delaunay triangulation consisting of 31 triangles (left) and 3-D Delaunay triangulation consisting of 6 tetrahedrons (right, two tetrahedrons are colored for clarity).
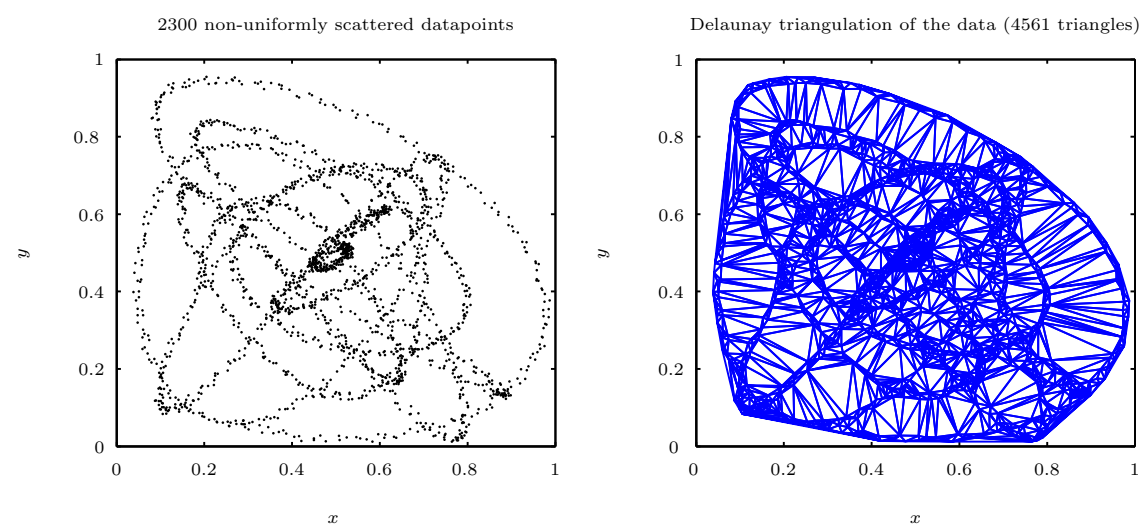

Fig. 4 Non-uniformly scattered dataset (left) and its direct Delaunay triangulation (right)
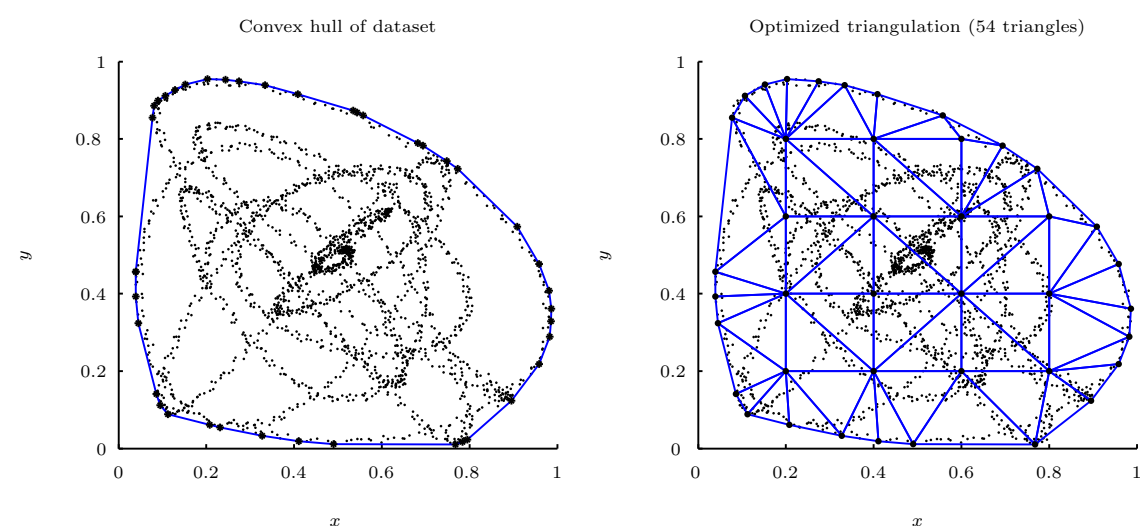

Fig. 5 Convex hull of dataset (left) and its well defined triangulation (right) 


\section{Spline Spaces}

A spline space is the space of all spline functions $s$ of a given degree $d$ and continuity order $C^{r}$ on a given triangulation $\mathcal{T}$. Such spline spaces have been studied extensively, see e.g. ${ }^{312426}$ We use the definition of the spline space from: ${ }^{26}$

$$
S_{d}^{r}(\mathcal{T}):=\left\{s \in C^{r}(\mathcal{T}):\left.s\right|_{t} \in \mathbb{P}_{d}, \forall t \in \mathcal{T}\right\}
$$

with $\mathbb{P}_{d}$ the space of all polynomials of total degree $d$. The definition of the spline space in Eq. (7) provides a convenient notation for stating the degree, continuity and triangulation of a spline solution without having to specify individual spline functions. For example, $S_{3}^{1}(\mathcal{T})$ is the space of all cubic spline functions with continuity order $C^{1}$ defined on the triangulation $\mathcal{T}$.

\section{The B-form of the multivariate simplex spline}

The simplex spline is a B-spline in the sense that it can be expressed in the well-known B-form. ${ }^{23}$ The B-form follows from the multinomial theorem:

$$
\left(b_{0}+b_{1}+\cdots+b_{n}\right)^{d}=\sum_{\kappa_{0}+\kappa_{1}+\cdots+\kappa_{n}=d} \frac{d !}{\kappa_{0} ! \kappa_{1} ! \cdots \kappa_{n} !} \prod_{i=0}^{n} b_{i}^{\kappa_{i}}
$$

At this point the multi-index $\kappa$ is introduced:

$$
\kappa:=\left(\kappa_{0}, \kappa_{1}, \ldots, \kappa_{n}\right) \in \mathbb{N}^{n+1}
$$

The 1-norm of the multi-index is given by:

$$
|\kappa|=\kappa_{0}+\kappa_{1}+\cdots+\kappa_{n}=d, \quad d \geq 0
$$

The multi-index provides a convenient mechanism for covering all possible integer permutations that sum up to a value $d$.

$\square$ Example: List all valid permutations of $\kappa$ for $|\kappa|=3$ and $n=2$. For $n=2$ we have $\kappa=\left(\kappa_{0}, \kappa_{1}, \kappa_{2}\right)$. The set of valid permutations of $\kappa$ are:

$$
\kappa \in\{(3,0,0),(2,1,0),(2,0,1),(1,2,0),(1,1,1),(1,0,2),(0,3,0),(0,2,1),(0,1,2),(0,0,3)\}
$$

$\mathrm{Hu}^{34}$ and $\mathrm{Lai}^{26}$ introduce a very useful lexicographical sorting order on the elements of the multi-index:

$$
\kappa_{d, 0,0 \cdots 0}>\kappa_{d-1,1,0 \cdots 0}>\kappa_{d-1,0,1,0 \cdots 0}>\cdots>\kappa_{0 \cdots 0,1, d-1}>\kappa_{0 \cdots 0,0, d}
$$

The total number of valid permutations of $\kappa$ is $\hat{d}$ :

$$
\hat{d}=\frac{(d+n) !}{n ! d !}
$$

Using the multi-index from Eq. (9) the multinomial equation Eq. (8) can be simplified into:

$$
\left(b_{0}+b_{1}+\cdots+b_{n}\right)^{d}=\sum_{|\kappa|=d} \frac{d !}{\kappa !} b^{\kappa}
$$

Example: Expand the multinomial expression for $n=2$ and $d=2$ using the multi-index $\kappa$.

In this case we have $\kappa=\left(\kappa_{0}, \kappa_{1}, \kappa_{2}\right)$ with permutations $\kappa \in\{(2,0,0),(1,1,0),(1,0,1),(0,2,0),(0,1,1),(0,0,2)\}$. The multinomial equation Eq. (13) then is:

$$
\begin{aligned}
\left(b_{0}+b_{1}+b_{2}\right)^{2} & =\sum_{|\kappa|=2} \frac{2 !}{\kappa !} b^{\kappa} \\
& =\frac{2 !}{2 ! 0 ! 0 !} b_{0}^{2} b_{1}^{0} b_{2}^{0}+\frac{2 !}{1 ! 1 ! 0 !} b_{0}^{1} b_{1}^{1} b_{2}^{0}+\frac{2 !}{1 ! 0 ! 1 !} b_{0}^{1} b_{1}^{0} b_{2}^{1}+\frac{2 !}{0 ! 2 ! 0 !} b_{0}^{0} b_{1}^{2} b_{2}^{0}+\frac{2 !}{0 ! 1 ! 1 !} b_{0}^{0} b_{1}^{1} b_{2}^{1}+\frac{2 !}{0 ! 0 ! 2 !} b_{0}^{0} b_{1}^{0} b_{2}^{2} \\
& =b_{0}^{2}+2 b_{0} b_{1}+2 b_{0} b_{2}+b_{1}^{2}+2 b_{1} b_{2}+b_{2}^{2}
\end{aligned}
$$


The basis function $B_{\kappa}^{d}(b)$ of the multivariate spline can now be defined as follows:

$$
B_{\kappa}^{d}(b):=\frac{d !}{\kappa !} b^{\kappa}
$$

De Boor proved ${ }^{23}$ that $\left\{B_{\kappa}^{d}(b), \kappa \in \mathbb{N}^{n+1},|\kappa|=d\right\}$ is a stable basis for the space of polynomials of degree $d$. This means that any polynomial $p(b)$ of degree $d$ can be written as a linear combination of $B_{\kappa}^{d}$ 's as follows:

$$
p(b)=\sum_{|\kappa|=d} c_{\kappa} B_{\kappa}^{d}(b)
$$

with $c_{\kappa}$ a vector of linear coefficients called control coefficients, or more commonly, B-coefficients. The subscript multi-index $\kappa$ is alternatively called the indexer of $c$. The B-form of the multivariate spline follows from Eq. (15):

$$
s(b)=\sum_{|\kappa|=d} c_{\kappa}^{t} B_{\kappa}^{d}(b), \quad b \in t \in \mathcal{T}
$$

The total number of B-coefficients for a $d$-th order basis function on an $n$-dimensional simplex is equal to the total number of valid permutations of $\kappa: \hat{d}$, with $\hat{d}$ given by Eq. (12). The B-form can be evaluated using with the de Casteljau algorithm from, ${ }^{34}$ or directly by simply expanding the B-form Eq. (16), which is computationally more efficient.

\section{E. The B-coefficient net}

The B-coefficients are strongly structured in what is called the B-coefficient net, or B-net for short. The B-net has a spatial representation that provides insight into the structure of the B-form. The B-net is also very useful in the visualization of the structure of continuity between simplices. The graphical representation of the B-net is well known in the literature, see e.g. ${ }^{26,32,35}$ In Fig. [6 the graphical representation of the B-net corresponding with a third degree basis function (i.e. $d=3$ ) defined on a triangulation consisting of the three simplices $t_{i}, t_{j}$ and $t_{k}$ is shown. There exists a direct relationship between the index of a B-coefficient and its spatial location, or barycentric coordinate within a simplex:

$$
b\left(c_{\kappa}\right)=\frac{\kappa_{0} v_{p_{0}}+\kappa_{1} v_{p_{1}}+\cdots+\kappa_{n} v_{p_{n}}}{d}, \quad|\kappa|=d
$$

with $b\left(c_{\kappa}\right)$ the barycentric coordinate of B-coefficients and $v_{p_{i}}, i=0,1, \ldots, n$ the simplex vertices.

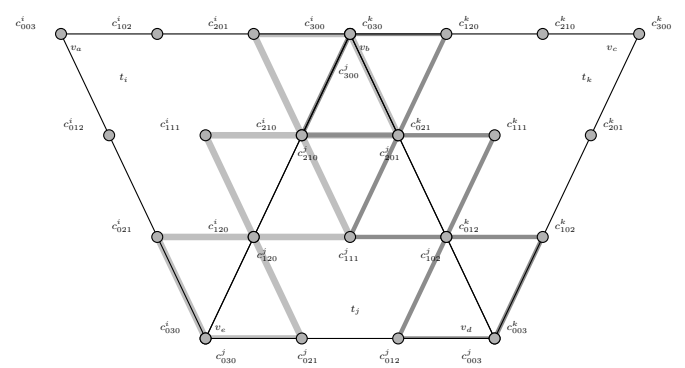

Fig. 6 B-net for $d=3$ basis function on 3 simplices together with $C^{1}$ continuity structure

\section{F. Continuity between Simplices}

A spline function is a piecewise defined polynomial function with $C^{r}$ continuity between its pieces. Continuity between the polynomial pieces is enforced by continuity conditions which are defined for every facet shared by two neighboring simplices. The formulation of the continuity conditions in this subsection are well known 
in the literature see e.g., ${ }^{23,25,26}$ but are repeated here for completeness. Let two neighboring $n$-simplices $t_{i}$ and $t_{j}$, differing by only the vertex $\sigma$, be defined as follows:

$$
t_{i}=\left\langle v_{0}, v_{1}, \ldots, v_{n-1}, \sigma\right\rangle, t_{j}=\left\langle v_{0}, v_{1}, \ldots, v_{n-1}, v_{n}\right\rangle
$$

Then $t_{i}$ and $t_{j}$ meet along the facet $\tilde{t}$ given by:

$$
\tilde{t}=t_{i} \cap t_{j}=\left\langle v_{0}, v_{1}, \ldots, v_{n-1}\right\rangle
$$

Clearly, $\tilde{t}$ is an $(n-1)$-simplex. As de Boor observed $i^{23}$ the facet simplex $\tilde{t}$ is indirectly defined by either one of the vertices $v_{n}$ and $\sigma$. This is an important observation, because it simplifies the implementation the algorithm for formulating the continuity equations.

We use the formulation for the continuity conditions from Awanou ${ }^{27}$ and Lai: ${ }^{26}$

$$
c_{\left(\kappa_{0}, \ldots, \kappa_{n-1}, m\right)}^{t_{i}}=\sum_{|\gamma|=m} c_{\left(\kappa_{0}, \ldots, \kappa_{n-1}, 0\right)+\gamma}^{t_{j}} B_{\gamma}^{m}(\sigma), \quad 0 \leq m \leq r
$$

with $\gamma=\left(\gamma_{0}, \gamma_{1}, \ldots, \gamma_{n}\right)$ a multi-index independent of $\kappa$.

In Fig. 6] the graphical interpretation of the $C^{1}$ continuity structure for a third order B-net on three simplices is drawn. This graphical interpretation is well known, see e.g. ${ }^{32,35}$ In total there are 6 continuity equations (i.e. 3 per edge), with which $C^{1}$ continuity is achieved across the two edges. It is now easy to check that the formulation Eq. (20) is valid only for the continuity between simplices $t_{i}$ and $t_{j}$ in figure Fig. 6 while it clearly fails to describe correct continuity structure between $t_{j}$ and $t_{k}$, as was noted in. ${ }^{29}$

In this case the formulation from Eq. (20) needs to be reformulated; the $m$ in the left multi-index and the 0 in the right multi-index need to be offset as follows:

$$
c_{\left(\kappa_{0}, m, \kappa_{2}\right)}^{i}=\sum_{|\gamma|=m} c_{\left(0, \kappa_{1}, \kappa_{2}\right)+\gamma}^{j} B_{\gamma}^{m}(\sigma), \quad 0 \leq m \leq r
$$

In general, the location of the constant multi-index value (i.e. the $m$ and the 0 ) is dependent on the location of the non-zero multi-index value in the indices of the out-of-edge vertices $v_{n}$ and $\sigma$.

For $C^{r}$ continuity there are a total of $R$ continuity conditions per edge facet:

$$
R=\sum_{m=0}^{r} \frac{(d-m+n-1) !}{(n-1) !(d-m) !}
$$

Eventually we want all continuity conditions for all facets formulated in the following matrix form:

$$
\mathbf{H c}=0
$$

Where matrix $\mathbf{H}$ is the so-called smoothness matrix. The vector $\mathbf{c}$ is the global vector of B-coefficients. Vector $\mathbf{c}$ is constructed as follows:

$$
\mathbf{c}=\left[\begin{array}{llll}
c_{\kappa}^{t_{1}} & c_{\kappa}^{t_{2}} & \ldots & c_{\kappa}^{t_{J}}
\end{array}\right]^{\mathbf{T}}
$$

Every $c_{\kappa}^{t_{i}}$ is sorted lexicographically as in Eq. (11). The matrix form of the continuity conditions follows from Eq. (20) by equating every continuity condition to zero. Every row in $\mathbf{H}$ thus corresponds with a single continuity condition Eq. (20). With $C^{r}$ continuity between simplices matrix $\mathbf{H}$ has size $(E \cdot R) \times(J \cdot \hat{d})$, with $E$ the total number of edges in a triangulation and $R$ and $\hat{d}$ as in Eq. (22) and Eq. (12) respectively. In general we have $\operatorname{rank}(\mathbf{H}) \leq(E \cdot R)$, but only for the simplest of triangulations will $\mathbf{H}$ be of full rank. The rank deficiency of $\mathbf{H}$ is caused by the fact that there are redundant continuity equations for triangulations with an interior vertex. ${ }^{26}$ For our purposes, we require $\mathbf{H}$ to be of full rank, that is, when $\mathbf{H}$ is a $R^{*} \times J \cdot \hat{d}$ matrix with $R^{*} \leq E \cdot R$ we have:

$$
\operatorname{rank} \mathbf{H}=R^{*}
$$

Our algorithm for constructing $\mathbf{H}$ therefore detects and removes any redundant continuity equations.

\section{Linear Regression with Simplex Splines}

In this section a brief introduction on linear regression with simplex splines will be given. The linear regression scheme presented here is was first introduced by de Visser, ${ }^{29}$ to which we would like to refer for a more complete coverage of the theory. 


\section{A. Preliminaries on linear regression with simplex splines}

Consider the pair of observations $(x(i), y(i))$ related as follows:

$$
y(i)=f(x(i))+r(i), \quad i=1,2, \ldots, N
$$

with $f$ an unknown function and with $r(i)$ a residual term. $\operatorname{In}^{29}$ a linear regression model structure for approximating $f$ is presented. This model structure is equivalent to a linear combination of B-form polynomials in $b(i)$, with $b(i)$ the barycentric coordinate of $x(i)$ with respect to the simplex $t_{j}$ as in Eq. (3). The B-form polynomials are of degree $d$ and defined on a triangulation consisting of $J$ simplices:

$$
y(i)=\sum_{j=1}^{J}\left(\delta_{j k(i)} \sum_{|\kappa|=d} c_{\kappa}^{t_{j}} B_{\kappa}^{d}(b(i))\right)+r(i)
$$

with $\delta_{j k(i)}$ the simplex membership operator defined as follows:

$$
\delta_{j k(i)}= \begin{cases}1, & \text { if } j=k(i) \\ 0, & \text { if } j \neq k(i)\end{cases}
$$

and with $k(i)$ an index function that produces the index of the simplex which contains the data point $x(i)$, i.e., $x(i) \in t_{k(i)}, \forall i$.

$\square$ Example: Define the regression model as a first degree multivariate spline function defined on two triangles. We then have for every observation:

$$
\begin{aligned}
y(i) & =\sum_{|\kappa|=1} c_{\kappa}^{t_{1}} B_{\kappa}^{1}(b(i))+\sum_{|\kappa|=1} c_{\kappa}^{t_{2}} B_{\kappa}^{1}(b(i))+\epsilon(i) \\
& =c_{100}^{t_{1}} b_{0}(i)+c_{010}^{t_{1}} b_{1}(i)+c_{001}^{t_{1}} b_{2}(i)+c_{100}^{t_{2}} b_{0}(i)+c_{010}^{t_{2}} b_{1}(i)+c_{001}^{t_{2}} b_{2}(i)+\epsilon(i)
\end{aligned}
$$

$\mathrm{In}^{29}$ a matrix formulation of the B-form for a triangulation consisting of $J$ simplices was presented. It was shown that a data-membership matrix operator must be defined to ensure that the matrix formulation is valid for the complete triangualation. For a single observation on the complete triangulation, this matrix operator was defined as follows:

$$
\mathbf{D}(i)=\left[\left(\mathbf{D}_{t_{j}}(i)\right)_{j, j}\right]_{j=1}^{J} \in \mathbf{R}^{(J \cdot \hat{d}) \times(J \cdot \hat{d})}
$$

in which the sub blocks $\mathbf{D}_{t_{j}}(i)$, located on the main diagonal of $\mathbf{D}(i)$, are defined as follows:

$$
\mathbf{D}_{t_{j}}(i)=\left[\left(\delta_{j, k(i)}\right)_{q, q}\right]_{q=1}^{\hat{d}} \in \mathbf{R}^{\hat{d} \times \hat{d}}
$$

with $\delta_{j, k(i)}$ the membership operator from Eq. (28). The full-triangulation basis function vector for a single observation is found using Eq. (32):

$$
\mathbf{B}^{d}(i)=\left[\begin{array}{llll}
\mathbf{B}_{t_{1}}^{d}(i) & \mathbf{B}_{t_{2}}^{d}(i) & \cdots & \mathbf{B}_{t_{J}}^{d}(i)
\end{array}\right] \in \mathbf{R}^{1 \times J \cdot \hat{d}}
$$

with $\mathbf{B}_{t_{j}}^{d}(i)$ the individual, lexicographically sorted basis function terms from Eq. (14):

$$
\mathbf{B}_{t_{j}}^{d}(i)=\left[B_{\kappa}^{d, t_{j}}(b(i))\right]_{|\kappa|=d} \in \mathbf{R}^{1 \times \hat{d}}
$$

The B-form of the multivariate simplex spline for the complete triangulation is the result of combining Eq. (24), Eq. (31) and Eq. (29):

$$
p(b(i))=\mathbf{B}^{d}(i) \cdot \mathbf{D}(i) \cdot \mathbf{c} \in \mathbf{R}^{1 \times 1}
$$

Now let $\mathbf{X}(i)$ be a single row in the full-triangulation regression matrix for all observations $\mathbf{X} \in \mathbf{R}^{N \times J \cdot \hat{d}}$ as follows:

$$
\mathbf{X}(i)=\mathbf{B}^{d}(i) \cdot \mathbf{D}(i) \in \mathbf{R}^{1 \times J \cdot \hat{d}}
$$

Returning to the linear regression model from Eq. (27), we then have for a single observation $y(i)$ :

$$
y(i)=\mathbf{X}(i) \mathbf{c}+r(i) \in \mathbf{R}^{1 \times 1}
$$

which, for all observations, leads to the well known formulation:

$$
\mathbf{y}=\mathbf{X c}+\mathbf{r} \in \mathbf{R}^{N \times 1}
$$




\section{B. A generalized least squares estimator for the B-coefficients}

Equation Eq. (36) can be solved using many different methods, depending on the assumptions made on the nature of the residual term $\mathbf{r}$. We will introduce a generalized least squares (GLS) estimator for Eq. (36), which implies the following assumptions on the residual $\mathbf{r}$ :

$$
E(\mathbf{r})=0, \quad \operatorname{Cov}(\mathbf{r})=\boldsymbol{\Sigma}
$$

with $\boldsymbol{\Sigma} \in \mathbf{R}^{N \times N}$ the residual covariance matrix, which is both nonsingular and positive definite. The well known (see e.g. ${ }^{36}$ ) GLS cost function is:

$$
J_{G L S}(\mathbf{c})=\frac{1}{2}(\mathbf{Y}-\mathbf{X} \mathbf{c})^{\top} \boldsymbol{\Sigma}^{-1}(\mathbf{Y}-\mathbf{X} \mathbf{c})
$$

Up to this point we have not discussed how continuity between simplices is achieved in the frame of the new regression scheme. As explained in Section $\mathrm{E}$ the continuity conditions are contained in the smoothness matrix $\mathbf{H}$ from Eq. (23). The continuity conditions act as constraints on B-coefficients located in the continuity structure of a triangulation. Therefore, the complete optimization problem can be stated as an equality constrained GLS problem (ECGLS) as follows:

$$
\min _{\mathbf{c}} J_{G L S}(\mathbf{c}), \text { subject to } \mathbf{H c}=0
$$

Using Lagrange multipliers this optimization problem can be formulated as a Karush-Kuhn-Tucker (KKT) system:

$$
\left[\begin{array}{cc}
\mathbf{X}^{\top} \boldsymbol{\Sigma}^{-1} \mathbf{X} & \mathbf{H}^{\top} \\
\mathbf{H} & 0
\end{array}\right]\left[\begin{array}{l}
\mathbf{c} \\
\nu
\end{array}\right]=\left[\begin{array}{c}
\mathbf{X}^{\top} \boldsymbol{\Sigma}^{-1} \mathbf{Y} \\
0
\end{array}\right]
$$

with $\nu$ vector of Lagrange multipliers. The coefficient matrix in Eq. (40) is the KKT matrix. The solution of the KKT system is:

$$
\left[\begin{array}{c}
\hat{\mathbf{c}} \\
\hat{\nu}
\end{array}\right]=\left[\begin{array}{ll}
\mathbf{C}_{1} & \mathbf{C}_{\mathbf{2}} \\
\mathbf{C}_{3} & \mathbf{C}_{4}
\end{array}\right] \cdot\left[\begin{array}{c}
\mathbf{X}^{\top} \boldsymbol{\Sigma}^{-1} \mathbf{Y} \\
0
\end{array}\right]
$$

with $\hat{\mathbf{c}}$ and $\hat{\nu}$ estimators for $\mathbf{c}$ and $\nu$ respectively. Rao shows in ${ }^{37}$ that the matrix in Eq. (41) is equal to the pseudoinverse of the KKT matrix:

$$
\left[\begin{array}{ll}
\mathbf{C}_{1} & \mathbf{C}_{2} \\
\mathbf{C}_{3} & \mathbf{C}_{4}
\end{array}\right]=\left[\begin{array}{cc}
\mathbf{X}^{\top} \boldsymbol{\Sigma}^{-1} \mathbf{X} & \mathbf{H}^{\top} \\
\mathbf{H} & 0
\end{array}\right]^{+}
$$

Note that the sizes of the submatrices $\mathbf{C}_{\mathbf{1}}, \mathbf{C}_{\mathbf{2}}$ and $\mathbf{C}_{\mathbf{3}}$ in Eq. (42) are equal to the sizes of $\mathbf{X}^{\top} \boldsymbol{\Sigma}^{-1} \mathbf{X}, \mathbf{H}^{\top}$ and $\mathbf{H}$ respectively.

\section{Aerodynamic Model Identification of the Cessna Citation II}

In this section the new multivariate simplex spline based identification method is used to create a global aerodynamic model of the Cessna Citation II laboratory aircraft operated by the Delft University of Technology and the Netherlands National Aerospace Laboratory (NLR) Fig. 1, The identification data was collected during $250+$ longitudinal and lateral flight test maneuvers, resulting in a dataset containing more than ten million data points. Two types of control inputs were used; short duration (i.e. 10s), high amplitude 3211 pulse sets and long term (i.e. $100 \mathrm{~s}$ ) semi-random sum of sines disturbance signals. The 3211 control inputs were executed automatically by the digital flight control computer integrated with the fly by wire control system of the laboratory aircraft. For the sum of sines control inputs, the pilots were required to keep the aircraft flying straight and level while the flight control computer excited the control surfaces with a semi-random sum of sines signal. In this case the pilots controlled the aircraft through a sidestick controller connected to the flight control computer. The control inputs from the pilots were superimposed on the disturbance signal, resulting in the actual inputs for the control surfaces.

Flight path reconstruction techniques based on an iterated extended Kalman filter (IEKF) were used to get a crisp estimation of aircraft state. ${ }^{38}$ The linear regression scheme for multivariate simplex splines 
from Sec. III was then used with the reconstructed aircraft state to identify spline based models for the non-dimensional aerodynamic force coefficients $C_{X}, C_{Z}$ and the non-dimensional aerodynamic moment coefficients $C_{l}, C_{m}$ and $C_{n}$. The identified spline models were validated with a subset of the flight test data with results from the validation showing a very close fit between model and reality.

\section{A. Derivation of dimensionless aerodynamic force and moment coefficients}

An aerodynamic model predicts aerodynamic forces and moments based on the aircraft state. In order to identify an aerodynamic model, an input-output dataset is required. In this case the input dataset consists of the aircraft state, which can be measured directly, while the output dataset consists of the aerodynamic forces and moments, which can not be measured directly. The aerodynamic forces and moments can be derived from the well-known aircraft equations of motion (see e.g. Stevens and Lewis ${ }^{3}$ ). Using the equations of motion, the total aerodynamic force coefficients $C_{X}, C_{Y}$ and $C_{Z}$ in the $F_{B}$ body-fixed reference system can be calculated:

$$
\begin{aligned}
C_{X} & =\frac{m}{\frac{1}{2} \rho V^{2} S} \cdot A_{x}, \\
C_{Y} & =\frac{m}{\frac{1}{2} \rho V^{2} S} \cdot A_{y}, \\
C_{Z} & =\frac{m}{\frac{1}{2} \rho V^{2} S} \cdot A_{z},
\end{aligned}
$$

with $m$ the aircraft mass, and with $A_{x}, A_{y}$ and $A_{z}$ the filtered and bias corrected specific aerodynamic forces along the axes of $F_{B}$.

The total aerodynamic moment coefficients $C_{L}, C_{M}$ and $C_{N}$ are given by:

$$
\begin{aligned}
C_{l} & =\frac{1}{\frac{1}{2} \rho V^{2} S b}\left(I_{x} \dot{p}-\left(I_{y}-I_{z}\right) q r-I_{x z}(\dot{r}+p q)\right), \\
C_{m} & =\frac{1}{\frac{1}{2} \rho V^{2} S \bar{c}}\left(I_{y} \dot{q}-\left(I_{z}-I_{x}\right) r p-I_{x z}\left(r^{2}-p^{2}\right)\right), \\
C_{n} & =\frac{1}{\frac{1}{2} \rho V^{2} S b}\left(I_{z} \dot{r}-\left(I_{x}-I_{y}\right) p q-I_{x z}(\dot{p}-q r)\right)
\end{aligned}
$$

with $p, q$ and $r$ the angular rates about the $F_{B} . I_{x}, I_{y}$, and $I_{z}$ are the products of inertia, while $I_{x z}$ is the single non-zero cross-product of inertia.

\section{B. Experiment setup}

Two different types of maneuvers were flown during the flight test campaign. With the first maneuver type, pilots were required to fly a disturbance rejection task. This type of maneuver leads to small angular rates, but high angular accelerations, and thus high values for the moment coefficients. The second maneuver type consisted of 3211 pulses on the control surfaces, and was executed automatically by the FBW control system. This type of maneuver is a classic method for obtaining high quality data for identification purposes (REF). In total, 39 parameters were measured and stored, resulting in a 39-dimensional parameter space see Table 1 The measurement rate for the parameters was $100 \mathrm{~Hz}$.

\section{Results from the flight testing}

In total, 184 disturbance rejection tasks and 763211 maneuvers were flown by 6 different pilots during eight test flights. Both longitudinal and lateral maneuvers were flown. The maneuvers were designed to cover a large part of the flight envelope of the Cessna Citation II. In Fig. 7 a 2-dimensional and 3-dimensional projection of the 39 dimensional parameter space is shown, together with a projection of the convex hull of the dataset. The convex hull of the dataset is a polyhedral subspace of the 39-dimensional parameter space which contains all data. Any point inside the convex hull of the dataset can be interpolated with a polynomial polynomial of at least degree one. In contrast, any point outside the convex hull of the dataset must be extrapolated. As such, the convex hull of the dataset plays an instrumental role in the identification 
Table 1 Measured parameters

\begin{tabular}{ll|ll}
\hline \hline parameters & description & parameter & description \\
\hline$t_{W D}[\mathrm{~s}]$ & time & $a_{x_{b}}\left[\mathrm{~m} / \mathrm{s}^{2}\right]$ & X-axis acceleration \\
$t[s]$ & maneuver time & $a_{y_{b}}\left[\mathrm{~m} / \mathrm{s}^{2}\right]$ & Y-axis acceleration \\
fofuD $[\mathrm{rad}]$ & forcing function & $a_{z_{b}}\left[\mathrm{~m} / \mathrm{s}^{2}\right]$ & Z-axis acceleration \\
fofuT $[\mathrm{rad}]$ & forcing function & $\mathrm{TAS}[\mathrm{m} / \mathrm{s}]$ & true airspeed \\
refRoll $[\mathrm{rad}]$ & roll reference & $\mathrm{IAS}[\mathrm{m} / \mathrm{s}]$ & indicated airspeed \\
refPitch $[\mathrm{rad}]$ & pitch reference & Mach $[-]$ & Mach number \\
RMSe $[\mathrm{rad}]$ & tracking error & $H_{p}[\mathrm{~m}]$ & pressure altitude \\
$\phi[\mathrm{rad}]$ & roll angle & $H_{b}[\mathrm{~m}]$ & calibrated altitude \\
$\theta[\mathrm{rad}]$ & pitch angle & $T_{\text {tot }}[\mathrm{K}]$ & total air temperature \\
$\psi[\mathrm{rad}]$ & yaw angle & $f \mathrm{ff}_{1}[\mathrm{~kg} / \mathrm{s}]$ & engine 1 fuel flow \\
$p[\mathrm{rad} / \mathrm{s}]$ & roll rate & $f \mathrm{~m}_{2}[\mathrm{~kg} / \mathrm{s}]$ & engine 2 fuel flow \\
$q[\mathrm{rad} / \mathrm{s}]$ & pitch rate & $x_{g p s}$ & GPS X-position \\
$r[\mathrm{rad} / \mathrm{s}]$ & yaw rate & $y_{g p s}$ & GPS Y-position \\
$a_{x}\left[\mathrm{~m} / \mathrm{s}^{2}\right]$ & X-axis acceleration & $z_{g p s}$ & GPS Z-position \\
$a_{y}\left[\mathrm{~m} / \mathrm{s}^{2}\right]$ & Y-axis acceleration & $v_{x_{g p s}}$ & GPS X-velocity \\
$a_{z}\left[\mathrm{~m} / \mathrm{s}^{2}\right]$ & Z-axis acceleration & $v_{y_{g p s}}$ & GPS Y-velocity \\
$\delta_{e}[\mathrm{rad}]$ & elevator deflection & $v_{z_{g p s}}$ & GPS Z-velocity \\
$\delta_{a}[\mathrm{rad}]$ & aileron deflection & & \\
$\delta_{r}[\mathrm{rad}]$ & rudder deflection & & \\
$\delta_{t_{e}}[\mathrm{rad}]$ & elevator trim & & \\
$\alpha[\mathrm{rad}]$ & angle of attack & & \\
$\beta[\mathrm{rad}]$ & angle of attack & & \\
\hline \hline
\end{tabular}

process as it determines the boundary between the space in which at least linear interpolation is possible, and the (far larger) space which can only be reached through extrapolation of existing data.

In order to obtain the true values for the aircraft states from the possibly noisy and biased sensor data, some form of flightpath reconstruction technique must be used. In our case, the procedure from Mulder et. al. ${ }^{38}$ based on an iterated extended Kalman filter was used to obtain crisp aircraft states.

In Fig. 8 an example is shown of a manually flown longitudinal disturbance rejection task, while Fig. 9 shows the results from a automatically flown longitudinal 3211 maneuver.

\section{Aerodynamic model structure selection}

The multivariate simplex spline based aerodynamic model consists of piecewise defined, ordinary polynomials with a predefined continuity between the polynomial pieces. Currently, the process of selecting polynomial terms is performed either by hand through expert knowledge, or through some advanced parameter selection scheme like that from Lombaerts et. al. ${ }^{13,39,40}$ In contrast with current methods, the aerodynamic model structure selection procedure for multivariate simplex spline based models is rather straightforward.

For the multivariate simplex spline based aerodynamic model, the model structure is defined as the complete spline space $S_{d}^{r}(\mathcal{T})$ (see Eq. (7)) of degree $d$ and continuity $r$ on the triangulation $\mathcal{T}$. This spline space contains all possible non-linear cross-couplings up to degree $d$ and has the same dimension as the triangulation $\mathcal{T}$.

The model selection process thus boils down to selection of the dimension of the spline space, selection of the maximum degree of the spline polynomials and the selection of the triangulation. Selection of the dimensions is relatively straightforward, and based on expert knowledge on the modeled system. The maximum possible dimension is, of course, limited by the total amount of measured parameters. Selection of the maximum degree of the spline polynomials is somewhat more complicated because, in general, it is hard to determine the maximum order of a complex system like an aircraft. Additionally, not all model dimensions have the same maximum order. For example, we could make the assumption that the influence of the angle of 

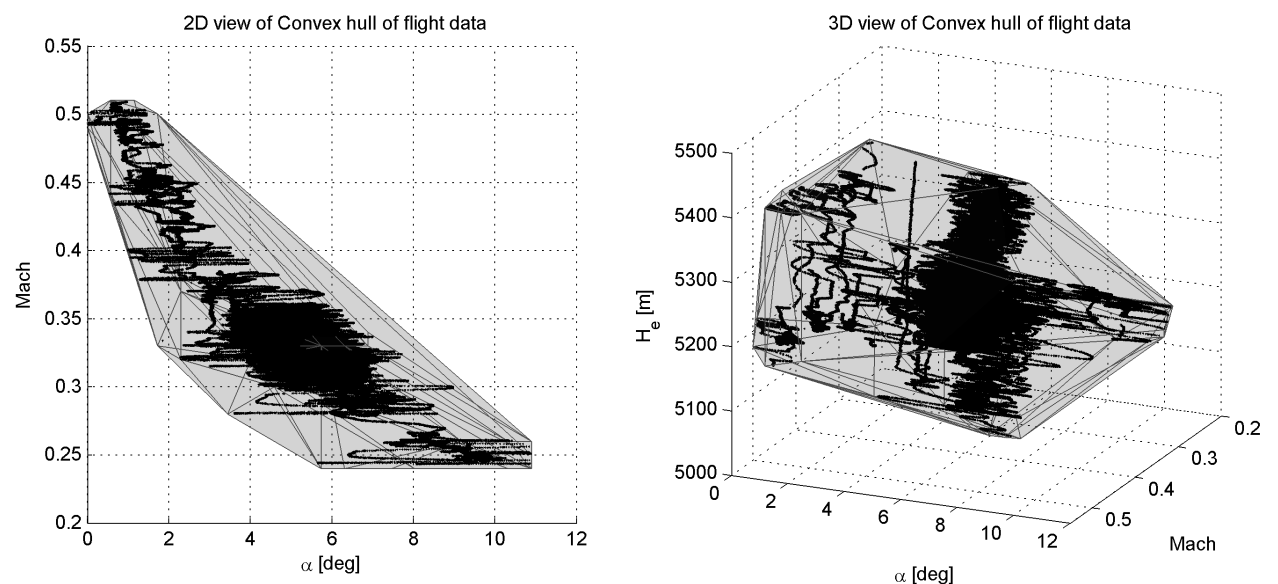

Fig. 7 2-D (left) and 3-D (right) projections of flight data, together with its convex hull
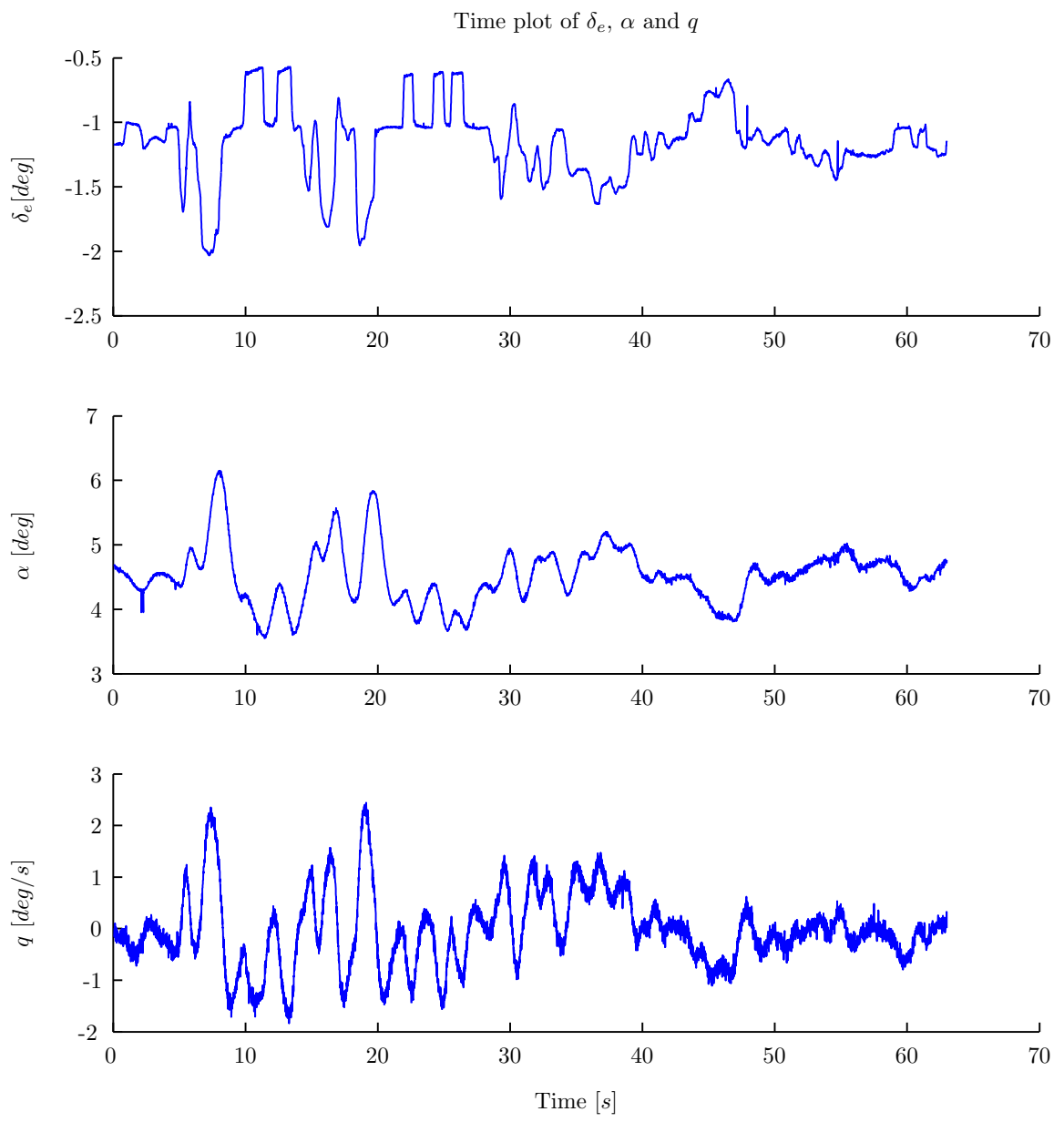

Fig. 8 Manually flown longitudinal disturbance rejection task 

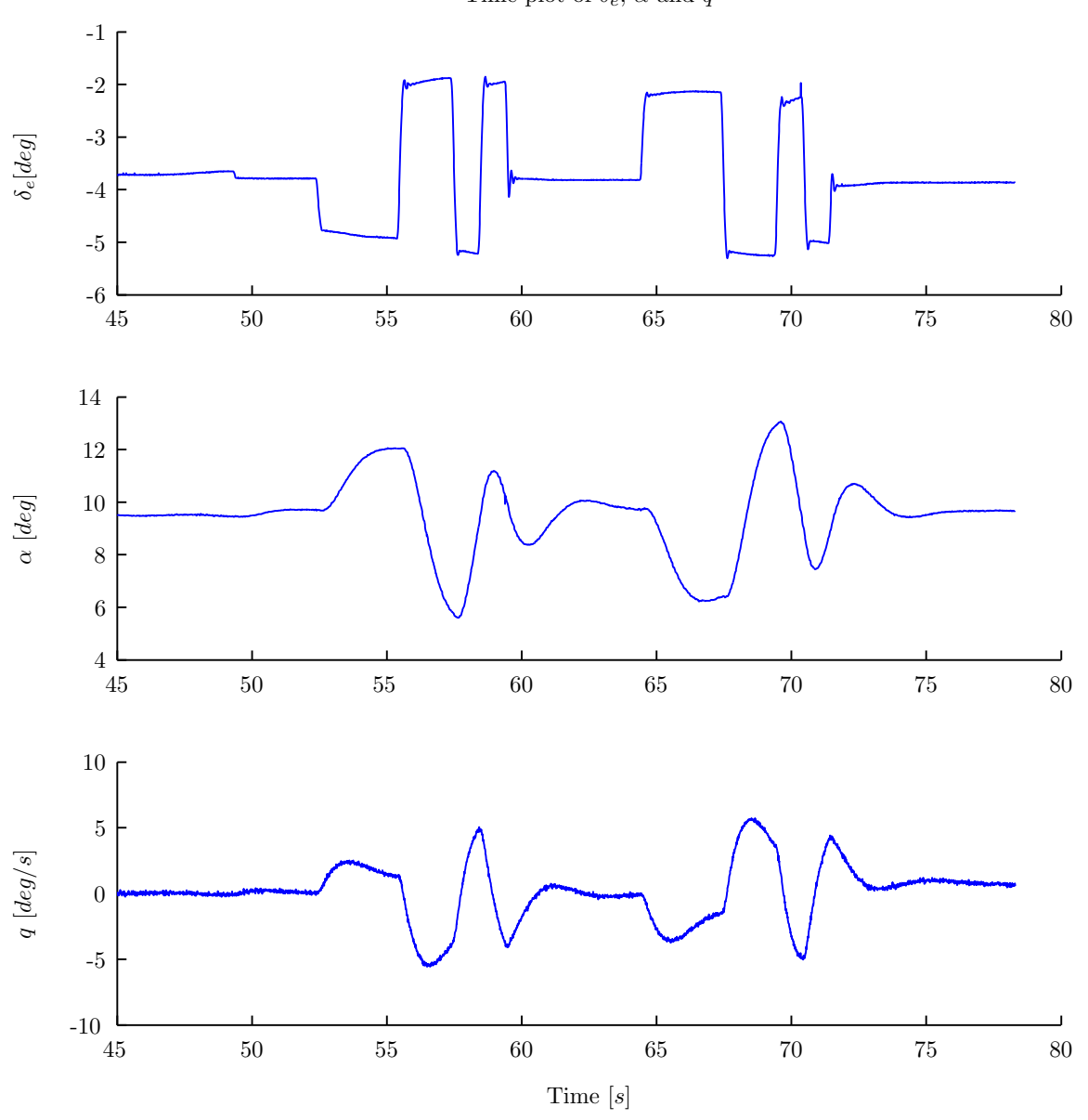

Fig. 9 Automatically executed 3211 elevator pulses 

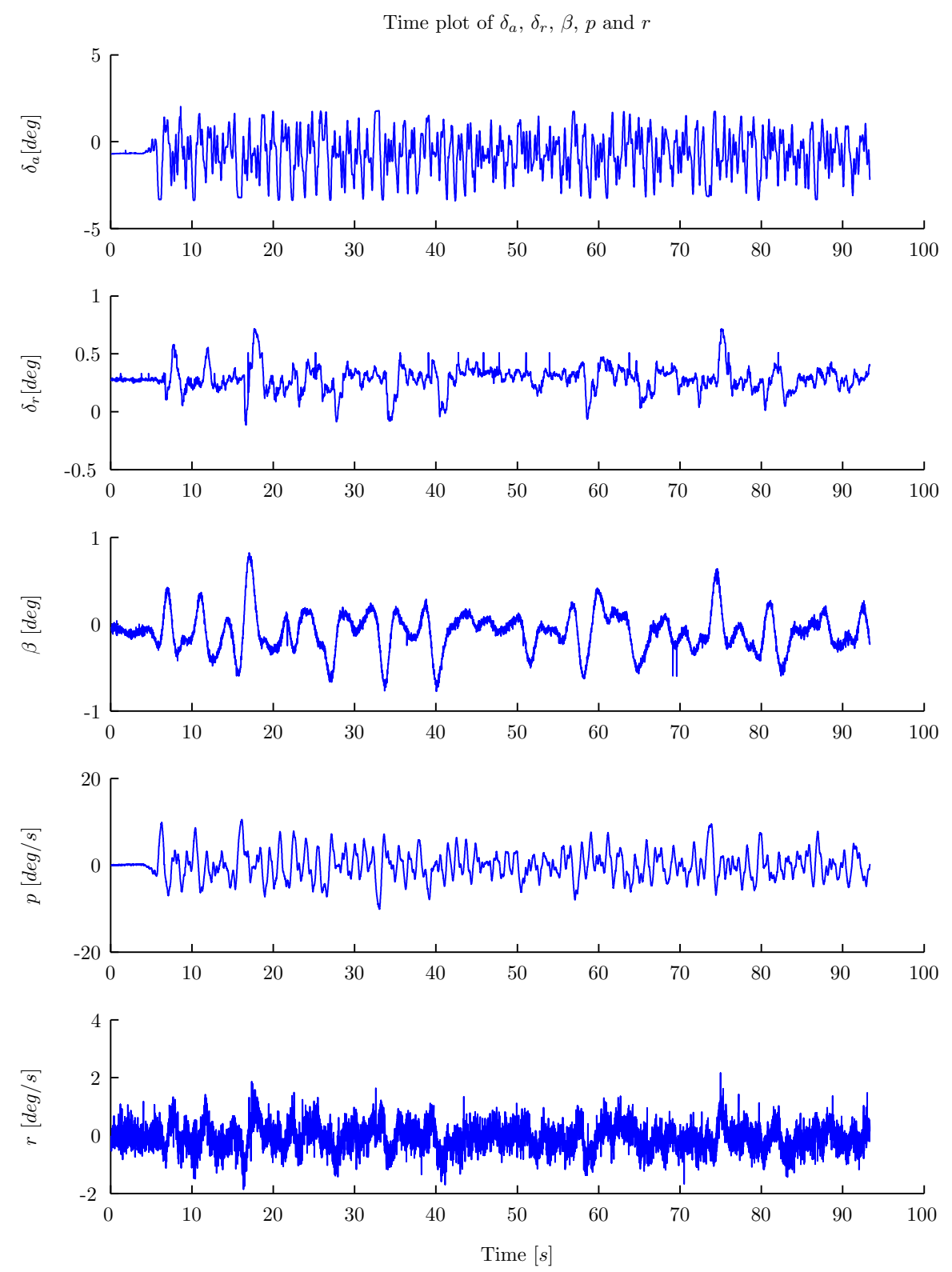

Fig. 10 Manually flown lateral disturbance rejection task 

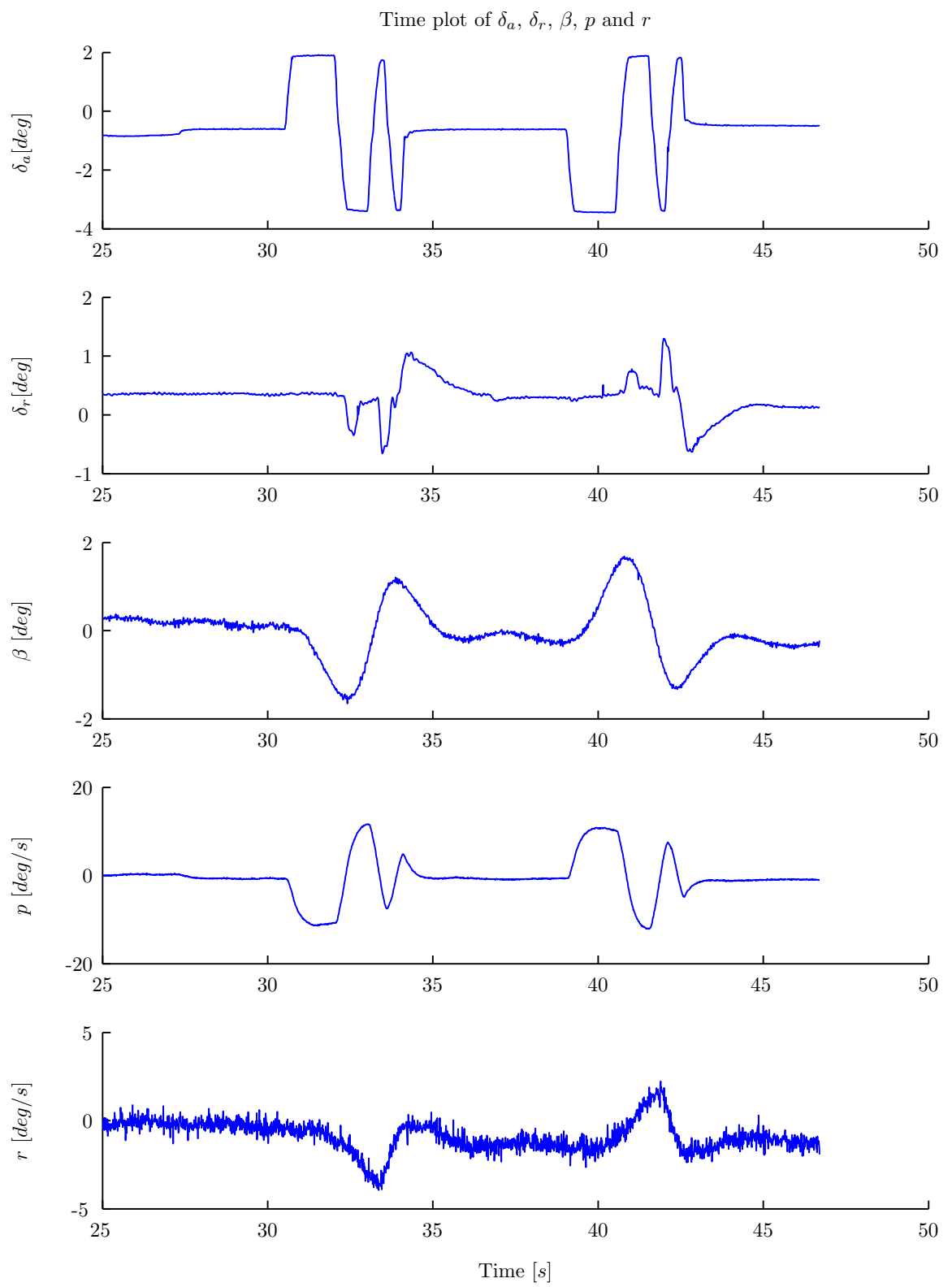

Fig. 11 Automatically executed 3211 aileron pulses 
attack $\alpha$ on the pitching moment coefficient $C_{m}$ is more complex than the influence on that same coefficient of the angle of sideslip $\beta$. Consequently, we expect the maximum degree of the spline polynomials in the dimension $\alpha$ to be higher than that of $\beta$. At first sight, this could pose a problem because the simplex spline polynomials are "isotropic", that is, the simplex spline polynomials have the same degree in all directions. This is not the case, however, because the coefficients are estimated using a least squares optimization, which is a convex optimization problem. ${ }^{41}$ Polynomial terms with a low physical significance will therefore have their coefficients set to zero, or close to zero, suppressing their influence.

The most complex task in selecting a model structure for a multivariate simplex spline based aerodynamic model is the determination of the triangulation $\mathcal{T}$. The triangulation is produced by partitioning the convex hull of the flight data (see e.g. Fig. 7) into a non-overlapping set of simplices, for example using the wellknown Delaunay triangulation method. Direct use of the Delaunay triangulation method will, in general, not result in a well defined triangulation. Such a triangulation will, for example, contain so called sliver simplices and simplices with too large or too small volumes. The initial triangulation of the convex hull of the dataset must therefore be optimized in some way, so as to produce a set of well defined simplices. Triangulation optimization is not a simple task, but advanced optimization algorithms for creating welldefined triangulations do exist. ${ }^{33,42,43}$ In our experience, however, we found that satisfactory triangulations could be generated using only basic optimization techniques, see Section [I].

The following model structures were used for the longitudinal aerodynamic model:

$$
\begin{aligned}
C_{X} & =f_{X}\left(\alpha, \delta_{e}, M\right) \in S_{3}^{1}\left(\mathcal{T}_{X}\right)+\epsilon_{X}, \\
C_{Z} & =f_{Z}\left(\alpha, \delta_{e}, M\right) \in S_{3}^{1}\left(\mathcal{T}_{Z}\right)+\epsilon_{Z}, \\
C_{m} & =f_{m}\left(\alpha, q, \delta_{e}\right) \in S_{3}^{1}\left(\mathcal{T}_{m}\right)+\epsilon_{m},
\end{aligned}
$$

with $f_{X}, f_{Z}$, and $f_{m}$ trivariate (i.e. 3 -dimensional) spline functions of degree 3 defined on their respective triangulations $\mathcal{T}_{X}, \mathcal{T}_{Z}$, and $\mathcal{T}_{m}$. The terms $\epsilon_{X}, \epsilon_{Z}$, and $\epsilon_{m}$ are residual terms, which should ultimately consist of zero-mean white noise.

The following model structures were selected for the lateral moment coefficients $C_{l}$ and $C_{n}$ :

$$
\begin{aligned}
C_{l} & =f_{l}\left(\alpha, \beta, \delta_{a}, p, r\right) \in S_{3}^{1}\left(\mathcal{T}_{l}\right)+\epsilon_{l}, \\
C_{n} & =f_{n}\left(\alpha, \beta, \delta_{r}\right) \in S_{3}^{1}\left(\mathcal{T}_{n}\right)+\epsilon_{n},
\end{aligned}
$$

with $f_{l}$ a 5 -variate and $f_{n}$ a trivariate spline functions of degree 3 defined on their respective triangulations $\mathcal{T}_{X}, \mathcal{T}_{Z}$, and $\mathcal{T}_{m}$. As with the longitudinal model structures, the terms $\epsilon_{l}$, and $\epsilon_{n}$ are the model residuals.

We should note that at this point, a model for the lateral force coefficient $C_{Y}$ could not be identified because of a lack of excitation in the lateral acceleration $A_{y}$. Therefore, additional flight test maneuvers designed specifically to excite $A_{y}$ will be performed in the near future.

In practice, the aerodynamic model structure selection was an iterative process. The process starts by selecting a set of model dimensions $\mathcal{D}$, organized in order of relevance. For example, if we are modeling $C_{m}$ then we expect $\alpha$ and $\delta_{e}$ to be of higher relevance than say $p$ or $\delta_{a}$. From this set of model dimensions, an active set is chosen with which the identification process is initialized. The identification process then starts by identifying the simplest possible model, that is, a first degree (linear) model defined on a single simplex. The degree of the spline polynomials is then increased by one, after which the performance of the model is compared with the performance of the lower degree. If performance has not increased, than the linear model is the highest degree model for the given triangulation. The resolution of the triangulation is then increased, after which the degree raising process of the previous step is repeated. If the residual is significantly large, and is not reduced by either degree raising or by increasing the triangulation resolution, then the set of spline function dimensions is assumed to be incomplete. In that case, a dimension from the list of relevant dimensions is added to the set of active dimensions. The process of degree raising and triangulation refinement is then repeated. If the residual is not reduced, then the added dimension is again removed from the active list. The optimization terminates when the desired performance levels have been reached, or the simplices in the triangulation have become so small that they do not contain enough data resulting in a singular KKT matrix. ${ }^{29}$

Our implementation of the identification algorithm is computationally efficient. For example, the estimation of the complete 5-dimensional model of degree 3 on 37 simplices for the aerodynamic rolling moment coefficient $C_{l}$ was based on 200000 data points and took little more than a second to complete on a single core of an Intel Q6600 2.4 Ghz processor. 


\section{E. Aerodynamic model identification \& validation}

Before starting the process of aerodynamic model identification, the simulated flight data was split into two parts: an identification dataset and a validation dataset. The identification dataset was used to estimate the parameters (i.e. the B-coefficients) of the multivariate simplex spline functions. The quality of the model was determined by comparing the output from the spline model with the measured force and moment coefficients using the validation dataset.

Two measures of performance were used to determine model quality; the root mean squared of the model error and the root mean squared of the relative model error. The root mean squared of the model error is given by:

$$
R M S_{C}=\sqrt{\frac{1}{N} \sum_{i=1}^{N} \epsilon_{C}(i)},
$$

with $\epsilon_{C}(i)$ the error between the coefficient $C$ and the spline model at the $i^{\text {th }}$ validation data point. The relative model error is a normalized performance measure given by:

$$
R R M S_{C}=\sqrt{\frac{1}{N} \sum_{i=1}^{N} \frac{\epsilon_{C}(i)}{|\max C-\min C|}}
$$

with $\min C$ and $\max C$ minimum and maximum value respectively of the measured aerodynamic coefficient $C$.

The least squares estimator from Eq. (41) was used to estimate the B-coefficients of the multivariate simplex splines. The measurement covariance matrix was set to $\Sigma=\mathbf{I}$, effectively reducing the generalized least squares estimator Eq. (41) into an ordinary least squares estimator.

In Table 2 the results of the validation are shown for the different multivariate spline based models. The numbers in the table clearly show that the models for $C_{Z}, C_{l}$ and $C_{m}$ are of very high quality with RRMS scores of less than $3 \%$. The models for $C_{n}$ and $C_{X}$ are of lower quality; especially the model for $C_{X}$ has a relatively low performance with a RRMS score of almost $7 \%$.

Table 2 Results of the model validation

\begin{tabular}{llccc}
\hline \hline Coefficient & Spline model & Simplices & error RMS & relative error RMS \\
\hline$C_{X}$ & $f_{X}\left(\alpha, \delta_{e}, M\right) \in S_{3}^{1}\left(\mathcal{T}_{X}\right)$ & 6 & $1.97 \mathrm{e}-002$ & $6.93 \%$ \\
$C_{Y}$ & no model & - & - & - \\
$C_{Z}$ & $f_{Z}\left(\alpha, \delta_{e}, M\right) \in S_{3}^{1}\left(\mathcal{T}_{Z}\right)$ & 6 & $3.01 \mathrm{e}-002$ & $2.60 \%$ \\
$C_{l}$ & $f_{l}\left(\alpha, \beta, \delta_{a}, p, r\right) \in S_{3}^{1}\left(\mathcal{T}_{l}\right)$ & 37 & $3.09 \mathrm{e}-004$ & $1.70 \%$ \\
$C_{m}$ & $f_{m}\left(\alpha, q, \delta_{e}\right) \in S_{3}^{1}\left(\mathcal{T}_{m}\right)$ & 12 & $1.84 \mathrm{e}-003$ & $1.72 \%$ \\
$C_{n}$ & $f_{n}\left(\alpha, \beta, \delta_{r}\right) \in S_{3}^{1}\left(\mathcal{T}_{n}\right)$ & 6 & $7.69 \mathrm{e}-004$ & $4.72 \%$ \\
\hline \hline
\end{tabular}

A better insight in the models themselves is obtained by directly inspecting their output, and their validation output. In Fig. 12 the direct model output from the $f_{X}\left(\alpha, \delta_{e}, M\right)$ spline function is shown. From the figure it is clear that the model for $C_{X}$ is not well defined at the edges of the flight envelope. This is a phenomenon that is directly related to an inadequate filling of simplices with data, especially along simplex edges. This phenomenon is amplified if the set of model dimensions is incomplete. The resulting model output is shown in Fig. 13, clearly showing the shortcomings of the model. In order to create a more adequate model for $C_{X}$ more data is required near the edges of the flight envelope, and the set of dimensions for $f_{X}$ should be changed.

Fig. 14 shows the direct model output from the $f_{Z}\left(a l p h a, \delta_{e}, M\right)$ spline function modeling $C_{Z}$. While this model suffers from the same data filling problem as the model for $C_{X}$, the resulting model is of much better quality. The reason for this is that the set of dimensions for the spline model $f_{Z}$ is adequate. The output of the $f_{Z}$ spline function is shown in Fig. 15 which clearly shows a close match between model and measured output.

The model output from the spline function $f_{m}\left(\alpha, q, \delta_{e}\right)$ modeling the pitching moment coefficient $C_{m}$ is shown in Fig. 16. This model is well defined, and produces a very close fit with the validation data Fig. 117. 

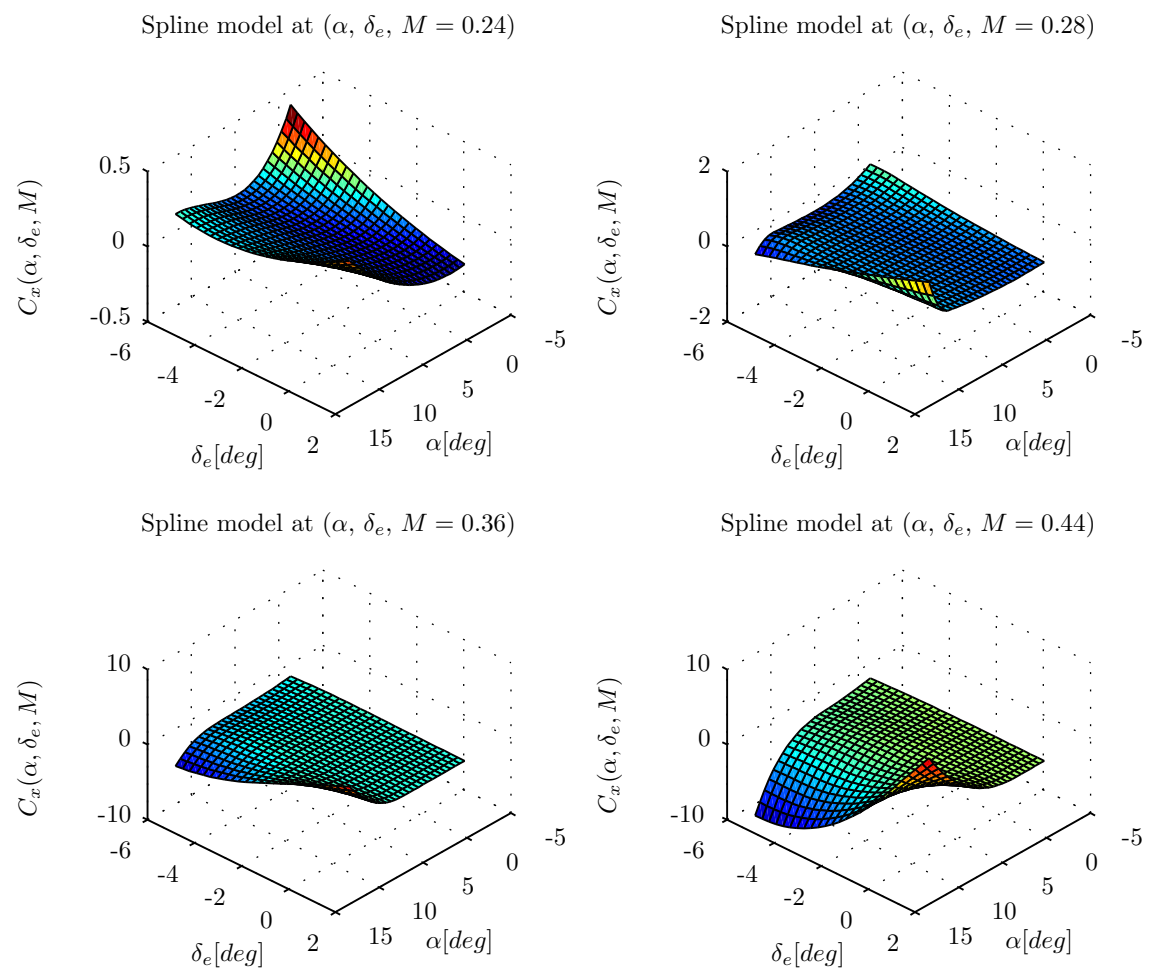

Fig. 12 Four 3-D slices through the global spline model $f_{X}\left(\alpha, \delta_{e}, M\right)$ for $C_{X}$ along the Mach axis

The model of $C_{m}$ shows some interesting facts. First, it is clear that $C_{m}$ is highly nonlinear along all model dimension. Second, the slope of the model for $C_{m}$ in the direction of $\alpha$ is observed to be negative in most flight conditions, that is, $C_{m_{\alpha}}<0$. This is, of course, as expected for a statically stable aircraft like the Cessna Citation II.

In Fig. 18 the direct model output from the $f_{l}\left(\alpha, \beta, \delta_{a}, p, r\right)$ spline function modeling $C_{l}$ is shown. The spline function $f_{l}$ is five dimensional function and is therefore also defined on a triangulation consisting of five dimensional simplices. The results from the model validation show a close fit between model and reality Fig. [19,

Finally, Fig. 20 shows the direct model output from the $f_{n}\left(\alpha, \beta, \delta_{r}\right)$ spline function. While the RRMS score of this model was only mediocre at $4.72 \%$, the model is actually quite well defined. The reason for the low RRMS score is made clear when observing the model validation results in Fig. 21] The measured values for $C_{n}$ are very noisy, and the excitation in $C_{n}$ is quite low.

\section{Conclusion}

In this paper, a new method for global aerodynamic model identification based on multivariate simplex splines is presented. This new identification method uses the B-form polynomials of multivariate simplex spline inside a novel linear regression framework. Standard parameter estimation techniques, like ordinary least squares, can then be used to estimate the B-coefficients of the multivariate simplex splines.

The new identification method was demonstrated on a set of flight data obtained during a flight test campaign with the Cessna Citation II laboratory aircraft operated by the Delft University of Technology and the Netherlands National Aerospace Laboratory. The flight data consisted of some 10 million data points collected during more than 250 flight test maneuvers. State of the art flight path reconstruction techniques based on an iterated extended Kalman filter were employed to reconstruct the aircraft states from the parameters measured during flight. Spline based models for the non-dimensional aerodynamic force and moment coefficients $C_{X}, C_{Z}, C_{l}, C_{m}$ and $C_{n}$ were then identified using the linear regression framework for 
Validation data (red) and Spline model output (blue) for $C_{x}$

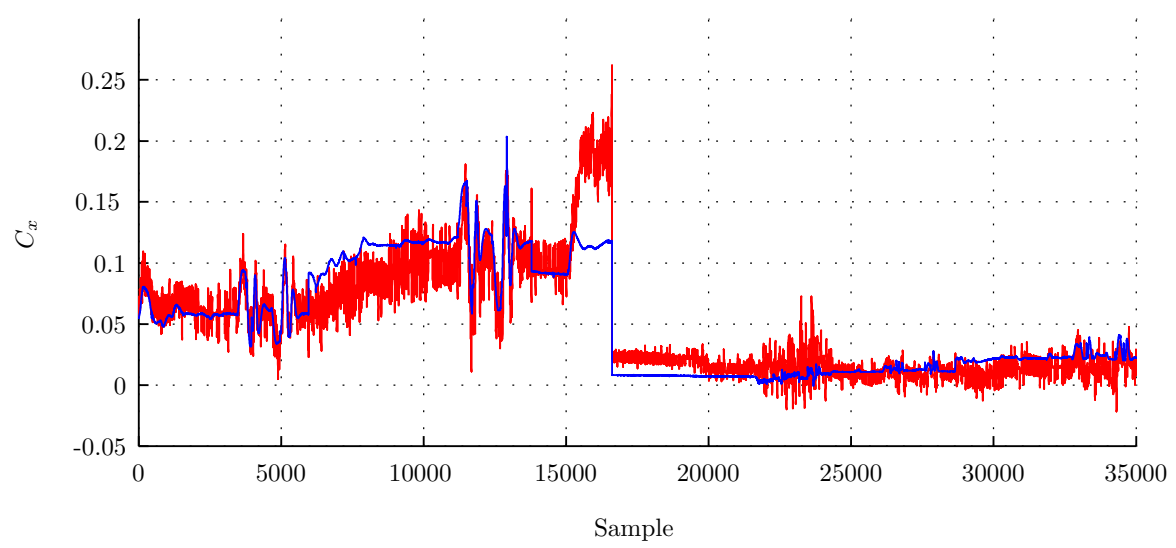

Spline model error for $C_{x}$

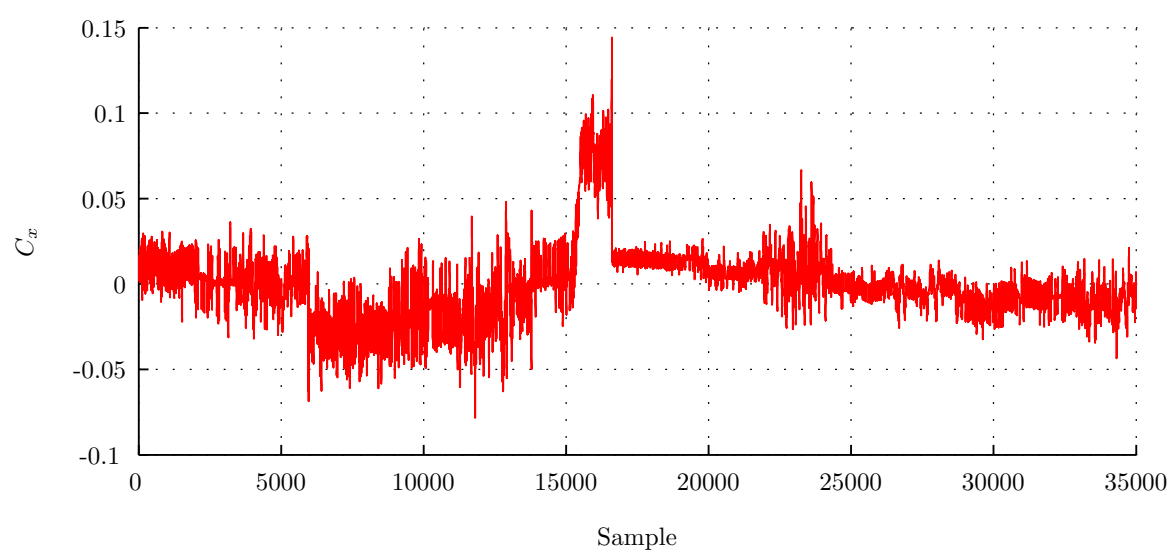

Fig. 13 Comparison between measured values for $C_{X}$ and model output from $f_{X}\left(\alpha, \delta_{e}, M\right)$ 

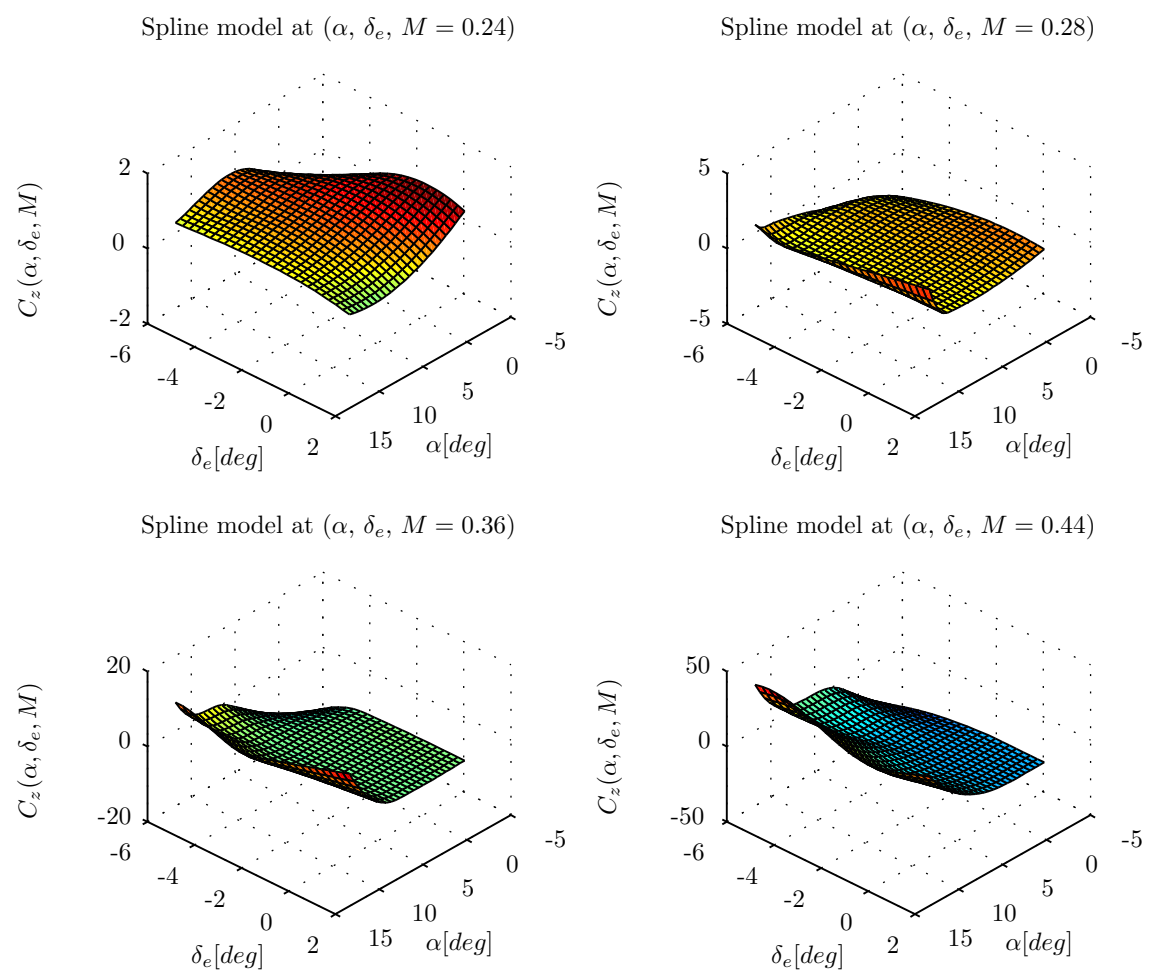

Fig. 14 Four 3-D slices through the global spline model $f_{Z}\left(\alpha, \delta_{e}, M\right)$ for $C_{Z}$ along the Mach axis

multivariate simplex splines.

The resulting spline models for the non-dimensional aerodynamic force and moment coefficients were validated with a subset of the flight data. Validation results showed that the spline based models for $C_{Z}$, $C_{m}$, and $C_{l}$ were of very high quality with relative error RMS values less than $3 \%$. The model for the yawing moment coefficient $C_{n}$ was of somewhat lower quality with a relative error RMS value of almost $5 \%$, but this was caused by the fact that the measured values for $C_{n}$ were very noisy and showed little excitation. The model for $C_{X}$, on the other hand, was more problematic. No combination of input dimensions could produce a model for $C_{X}$ that was in the same quality range as the other models. We hypothesize that this is caused by an inadequate filling of simplices with flight data, which causes the spline polynomials to diverge near the edges of the flight envelope.

The demonstration of the new identification method proves that multivariate simplex splines are a powerful tool for global nonlinear aerodynamic modeling. When gathering flight data for use with the new identification method, special care has to be taken to produce a dataset that adequately covers the region of the flight envelope for which a model is to be identified. Divergent behavior can be expected of spline polynomials defined at regions within the flight envelope with a low data density.

\section{References}

${ }^{1}$ Oelker, H., Renzo, B., Hoare, G. T., and Garcia-Mesuro, G., "Experiences on Aerodynamic Parameters Identification for Eurofighter at Daimler-Benz Aerospace, Alenia, British Aerospace and CASA," RTO SCI symposium on System Identification for Integrated Aircraft Development and Flight Testing, 1998.

${ }^{2}$ Oezger, E. and Meyer, E., "Aerodynamic Model Validation at EADS Military Aircraft," DGLR Conference, 2005.

${ }^{3}$ Stevens, B. L. and Lewis, F. L., Aircraft Control and Simulation, John Wiley \& Sons, Inc., 2003.

${ }^{4}$ Iliff, K. W., Maine, R., and Montgomery, T., "Important Factors in the Maximum Likelihood Analysis of Flight Test Maneuvers," Tecnical Paper 1459, NASA, 1979.

${ }^{5}$ Klein, V., Batterson, J. G., and Murphy, P. C., "Determination Of Aircraft Model Structure From Flight Data By Using Modified Stepwise Regression," Tech. Rep. 1916, NASA, 1981. 


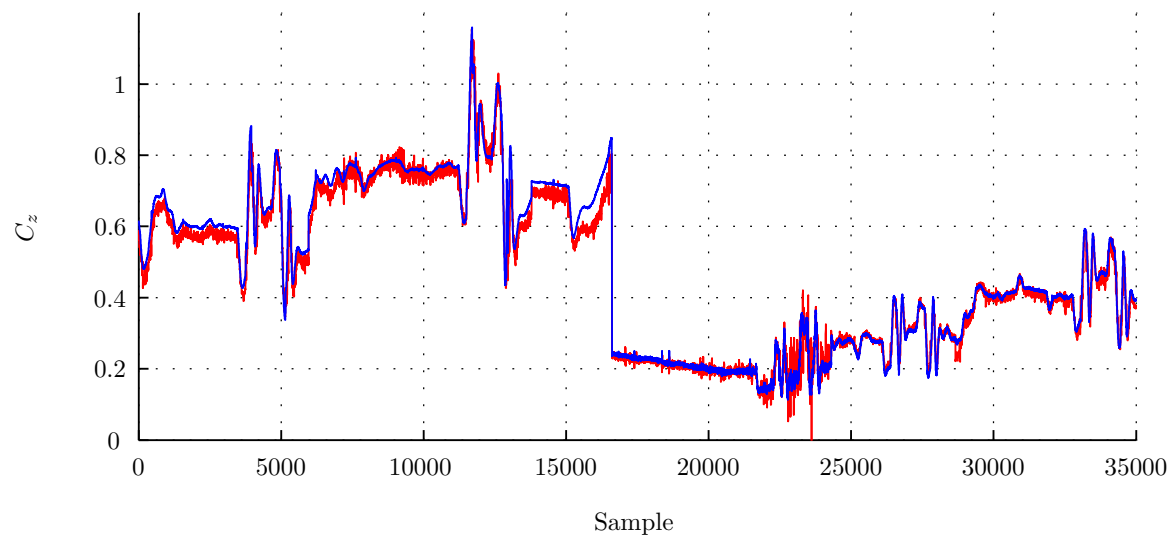

Spline model error for $C_{z}$

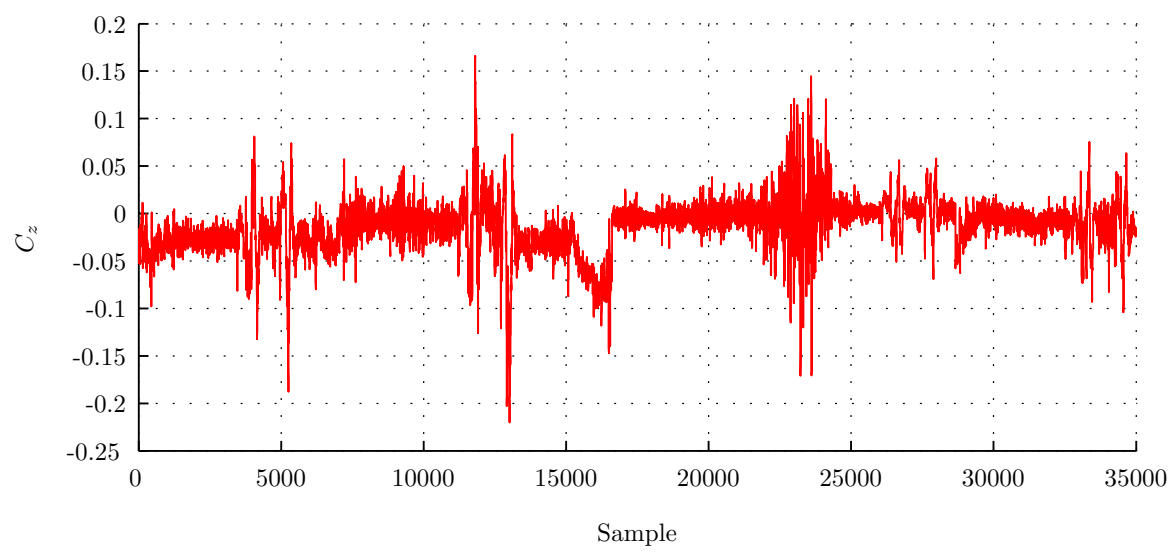

Fig. 15 Comparison between measured values for $C_{Z}$ and model output from $f_{Z}\left(\alpha, \delta_{e}, M\right)$ 

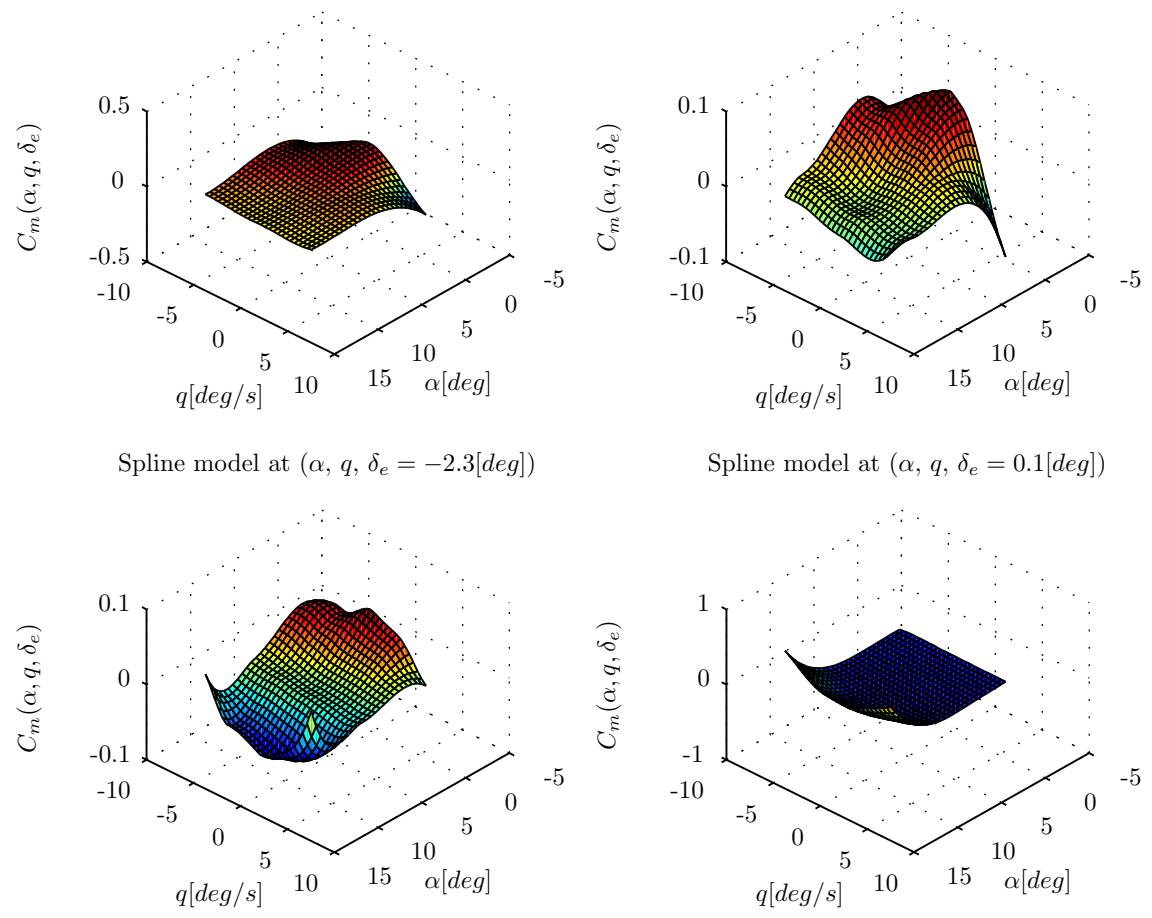

Fig. 16 Four 3-D slices through the global spline model $f_{m}\left(\alpha, q, \delta_{e}\right)$ for $C_{m}$ along the $\delta_{e}$ axis

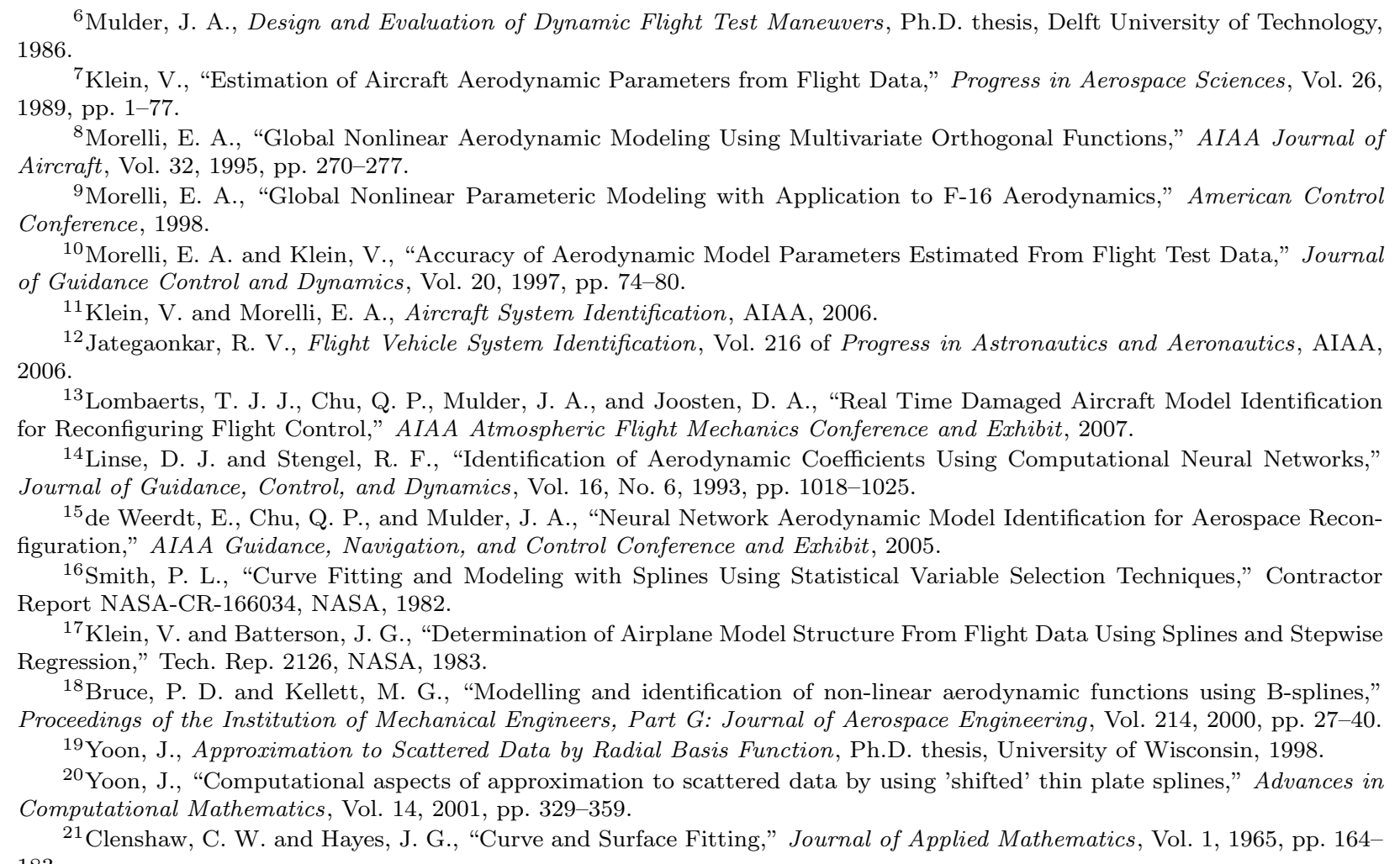
183. 


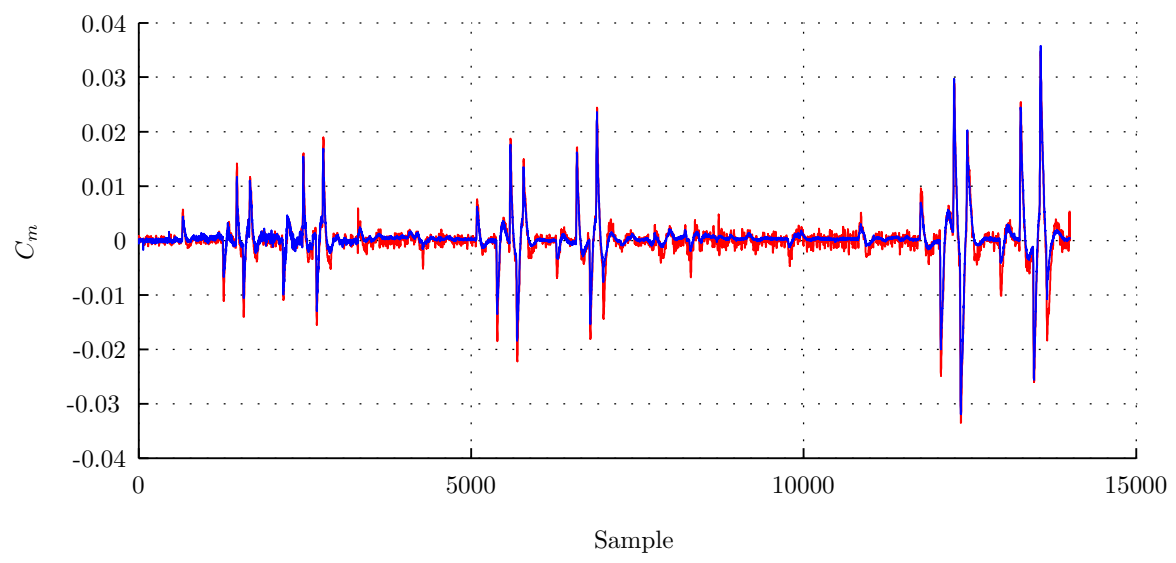

Spline model error for $C_{m}$

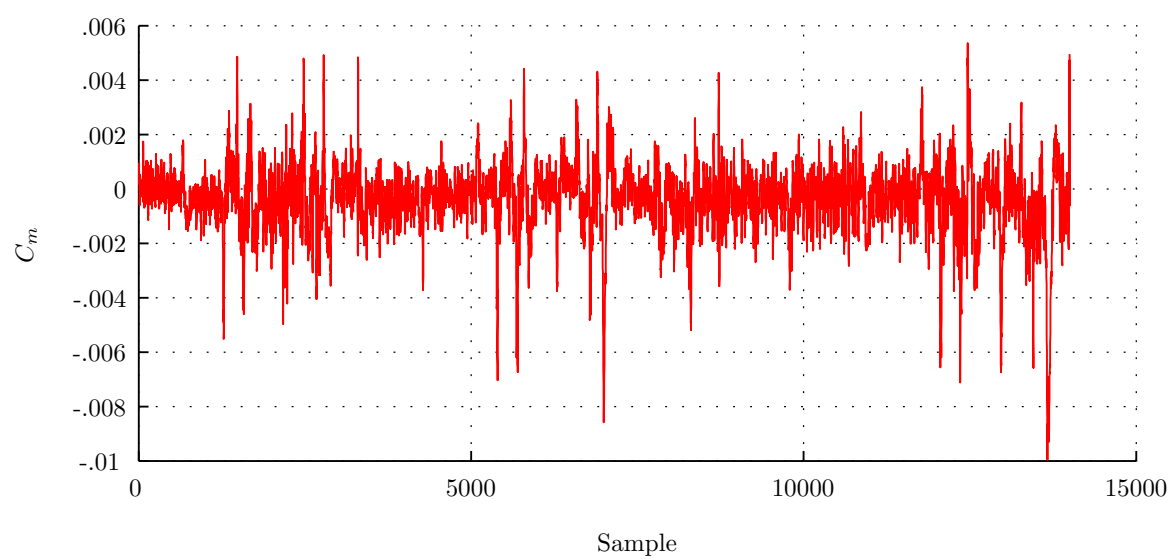

Fig. 17 Comparison between measured values for $C_{m}$ and model output from $f_{m}\left(\alpha, q, \delta_{e}\right)$ 

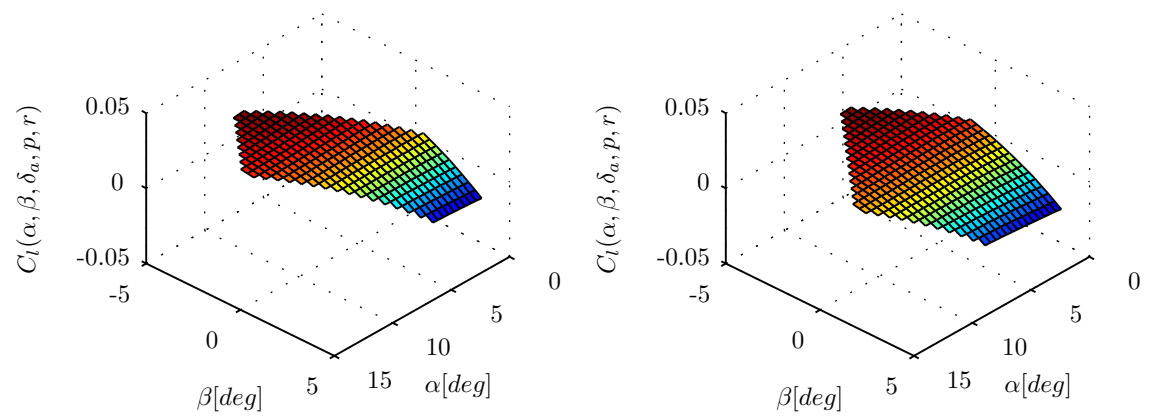

Spline model at $\left(\alpha, \beta, \delta_{a}=0.6[\mathrm{deg}], p, r\right)$

Spline model at $\left(\alpha, \beta, \delta_{a}=2.3[\mathrm{deg}], p, r\right)$
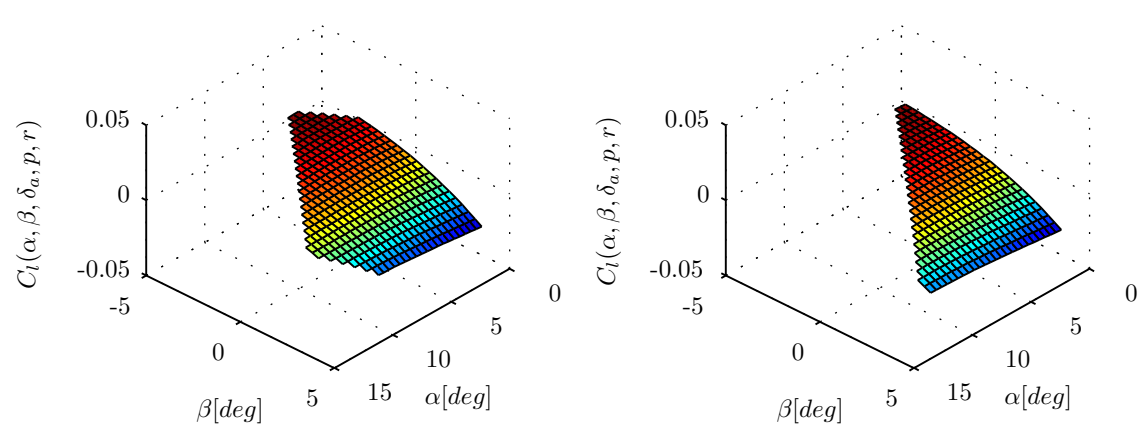

Fig. 18 Four 3-D slices through the global spline model $f_{l}\left(\alpha, \beta, \delta_{a}, p, r\right)$ for $C_{l}$ along the $\delta_{a}$ axis, and with $p$ and $q$ both at their minimum $-16[\mathrm{deg} / \mathrm{s}]$. 


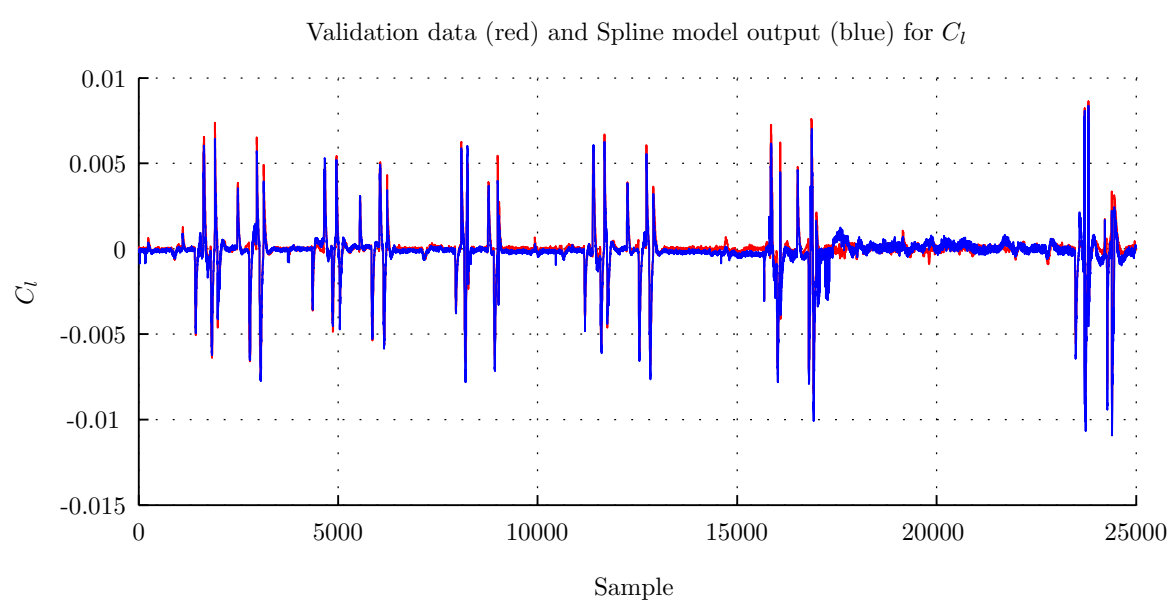

Spline model error for $C_{l}$

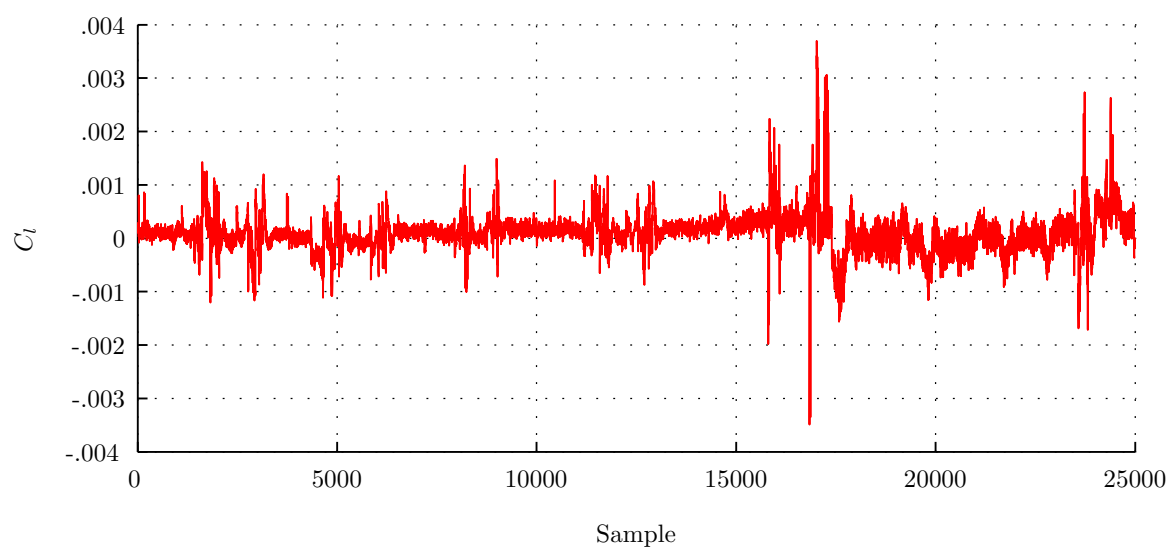

Fig. 19 Comparison between measured values for $C_{l}$ and model output from $f_{l}\left(\alpha, \beta, \delta_{a}, p, r\right)$ 

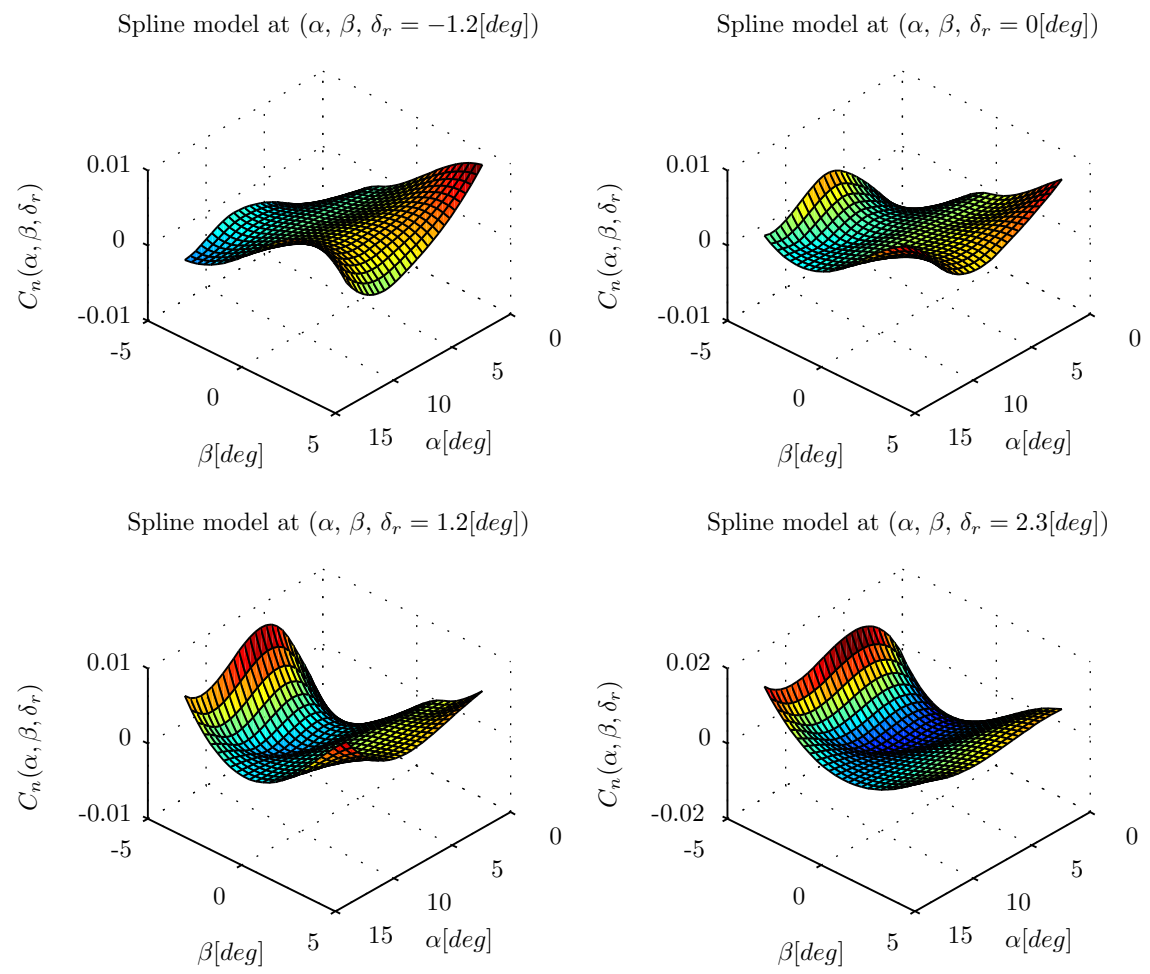

Fig. 20 Four 3-D slices through the global spline model $f_{n}\left(\alpha, \beta, \delta_{r}\right)$ for $C_{n}$

\footnotetext{
${ }^{22}$ Anderson, I. J., Cox, M. G., and Mason, J. C., "Tensor-Product Spline Interpolation to Data On or Near a Family of Lines," Numerical Algorithms, Vol. 5, 1993, pp. 193-204.

${ }^{23}$ de Boor, C., "B-form Basics," Geometric modeling: algorithms and new trends, edited by G. Farin, SIAM, 1987.

${ }^{24}$ Lai, M. J. and Schumaker, L. L., "On the Approximation Power of Bivariate Splines," Advances in Computational Mathematics, Vol. 9, 1998, pp. 251-279.

${ }^{25}$ Awanou, G., Energy Methods in 3D spline approximations of the Navier-Stokes Equations, Ph.D. thesis, University of Georgia, 2003.

${ }^{26}$ Lai, M. J. and Schumaker, L. L., Spline Functions over Triangulations, Cambridge University Press, 2007.

${ }^{27}$ Awanou, G., Lai, M. J., and Wenston, P., "The Multivariate Spline Method for Scattered Data Fitting and Numerical Solutions of Partial Differential Equations," Wavelets and Splines, 2005.

${ }^{28}$ Lai, M. J., "Multivariate Splines for Data Fitting and Approximation," 12th Approximation Theory Conference, 2007.

${ }^{29}$ de Visser, C. C., Chu, Q. P., and J. A. Mulder, J. A., "A New Approach to Linear Regression with Multivariate Splines," Automatica, Vol. 45, No. 12, 2009, pp. 2903-2909.

${ }^{30}$ de Visser, C., Mulder, J., and Chu, Q., "Global Nonlinear Aerodynamic Model Identification with Multivariate Splines," AIAA Atmospheric Flight Mechanics Conference, 2009.

${ }^{31}$ Lai, M., "Some Sufficient Conditions For Convexity of Multivariate Bernstein-B'ezier Polynomials and Box Spline Surfaces," Studia Scient. Math. Hung., Vol. 28, 1990, pp. 363-374.

${ }^{32}$ Lai, M. J., "Geometric Interpretation of Smoothness Conditions of Triangular Polynomial Patches," Computer Aided Geometric Design, Vol. 14, 1997, pp. 191-199.

${ }^{33}$ Ruppert, J., "A Delaunay Refinement Algorithm for Quality 2-Dimensional Mesh Generation," Journal of Algorithms, Vol. 18, 1995, pp. 548-585.

${ }^{34} \mathrm{Hu}$, X. L., Han, D. F., and Lai, M. J., "Bivariate Splines of Various Degrees for Numerical Solution of Partial Differential Equations," SIAM Journal on Scientific Computing, Vol. 29, 2007, pp. 1338-1354.

${ }^{35}$ Farin, G., "Triangular Bernstein-Bézier Patches," Computer Aided Geometric Design, Vol. 3, 1986, pp. 83-127.

${ }^{36}$ Kariya, T. and Kurata, H., Generalized Least Squares, John Wiley \& Sons, Inc., 2004.

${ }^{37}$ Rao, C. R., Linear Statistical Inference and Its Applications, John Wiley \& Sons, Inc., 2002.

${ }^{38}$ Mulder, J. A., Chu, Q. P., Sridhar, J. K., Breeman, J. H., and Laban, M., "Non-Linear Aircraft Flight Path Reconstruction Review and New Advances," Progress in Aerospace Sciences, Vol. 35, 1999, pp. 673-726.

${ }^{39}$ Lombaerts, T. J. J., Huisman, H. O., Chu, Q. P., Mulder, J. A., and Joosten, D. A., "Flight Control Reconfiguration based on Online Physical Model Identification and Nonlinear Dynamic Inversion," AIAA Guidance, Navigation and Control Conference and Exhibit, 2008.
} 


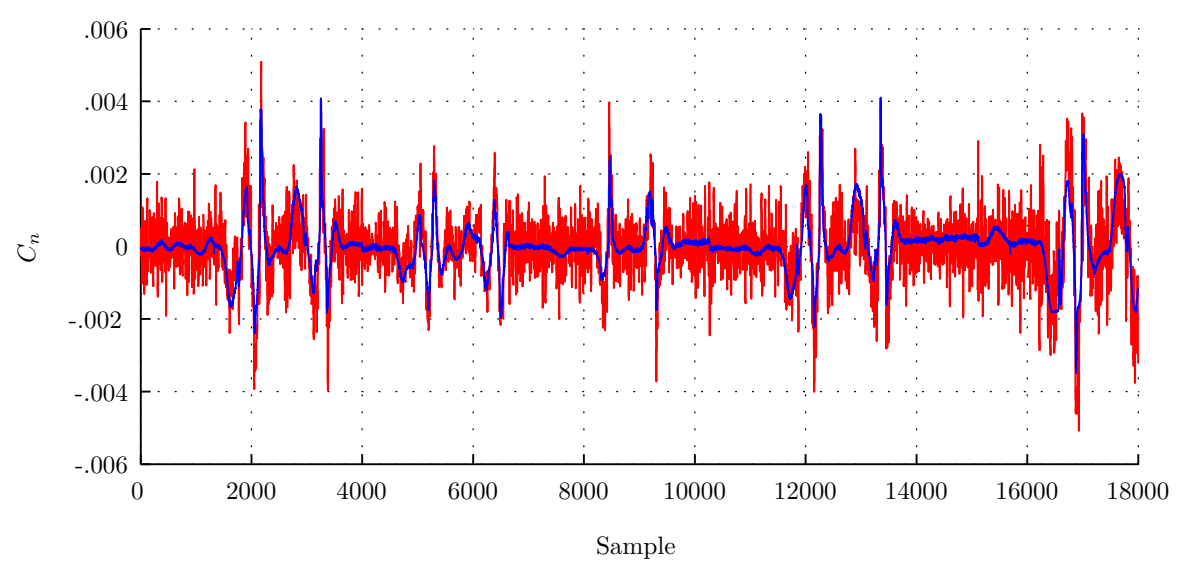

Spline model error for $C_{n}$

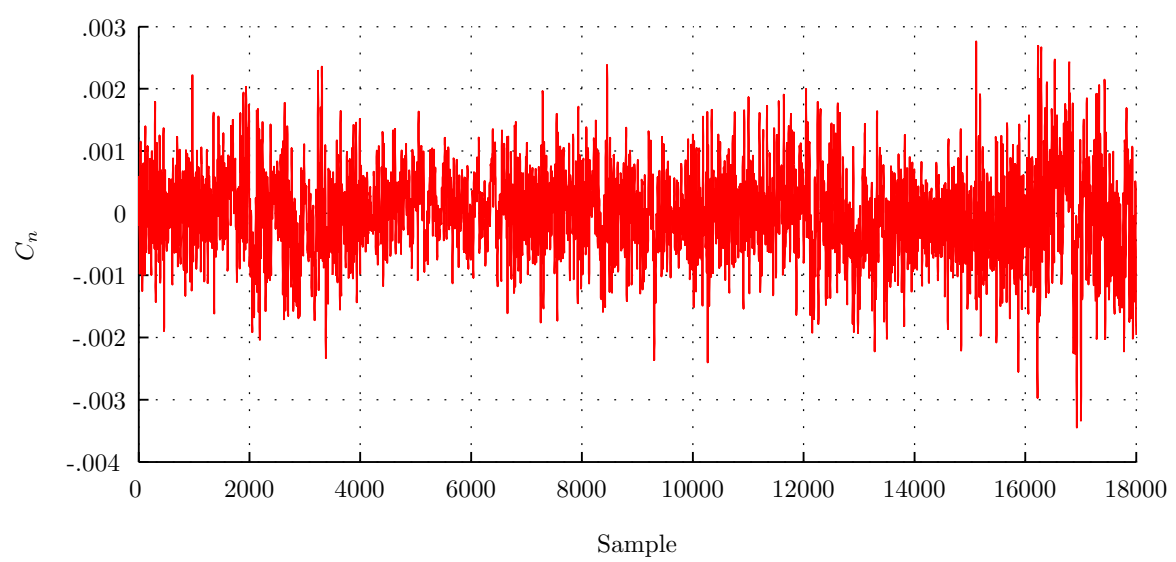

Fig. 21 Comparison between measured values for $C_{n}$ and model output from $f_{n}\left(\alpha, \beta, \delta_{r}\right)$ 
${ }^{40}$ Lombaerts, T. J. J., Huisman, H. O., Chu, Q. P., Mulder, J. A., and Joosten, D. A., "Nonlinear Reconfiguring Flight Control Based on Online Physical Model Identification," Journal Of Guidance, Control, and Dynamics, Vol. 32, 2009, pp. 727748.

${ }^{41}$ Boyd, S. P. and Vandenberghe, L., Convex Optimization, Cambridge University Press, 2004.

${ }^{42}$ Shewchuk, J. R., "Delaunay Refinement Algorithms for Triangular Mesh Generation," Computational Geometry, Vol. 22, 2001 , pp. 21-74.

${ }^{43}$ de Weerdt, E., de Visser, C. C., Chu, Q. P., and Mulder, J. A., "Fuzzy Simplex Splines," IFAC SYSID 2009, 2009. 